\title{
REACTIVE TRANSPORT MODELING OF AQUEOUS ALTERATION IN THE MURRAY FORMATION, GALE CRATER, MARS
}

Elisabeth M. Hausrath ${ }^{1, *}$, Douglas W. Ming ${ }^{2}$, Elizabeth B. Rampe ${ }^{2}$, and Tanya S. Peretyazhko ${ }^{3}$

${ }^{1}$ University of Nevada, Las Vegas, 4505 S. Maryland Parkway, Las Vegas, NV 89154

Elisabeth.Hausrath@unlv.edu, ${ }^{2}$ NASA Johnson Space Center, 2101 E. NASA Parkway Houston,

TX $77058{ }^{3}$ Jacobs, NASA Johnson Space Center, 2101 E. NASA Parkway Houston TX 77058 

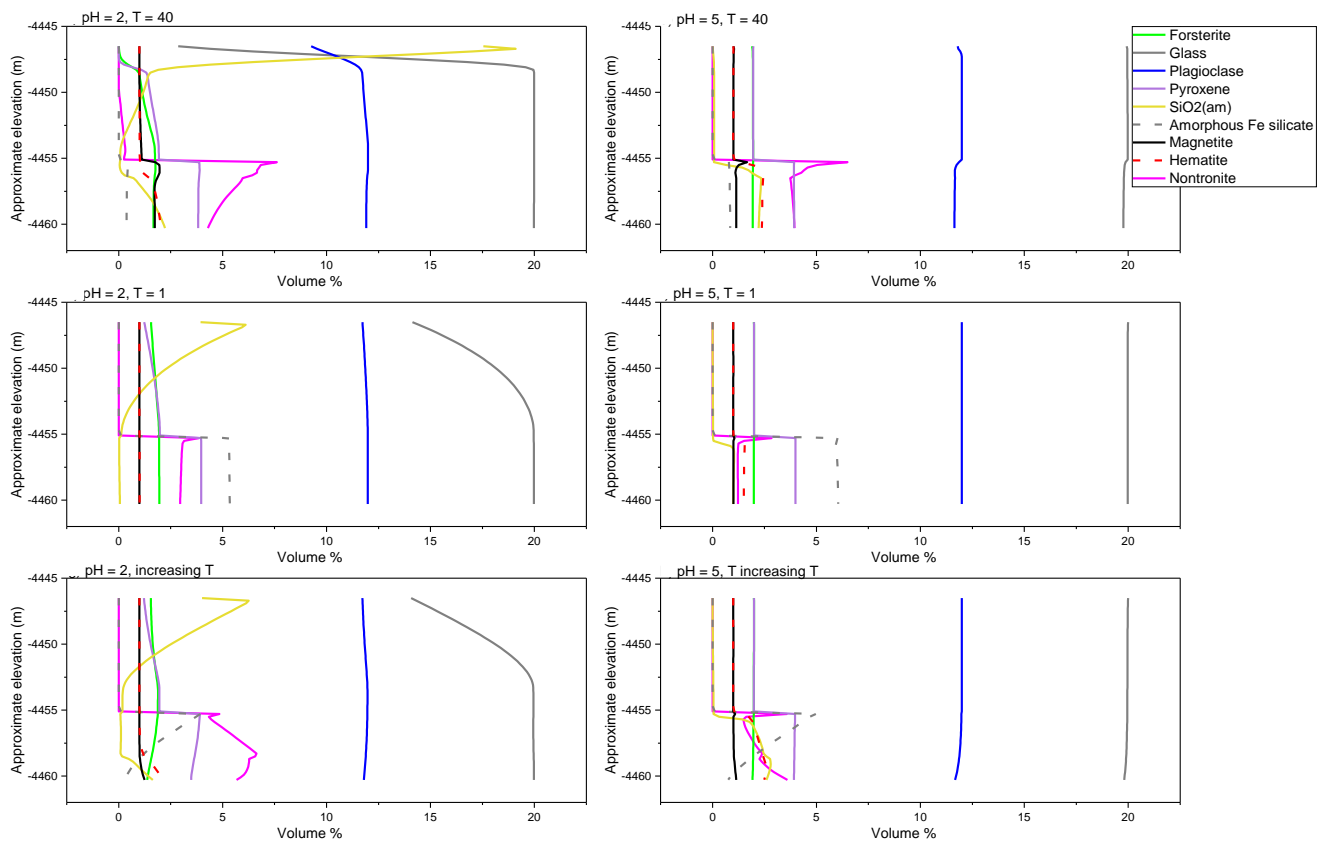

Figure S1. Figure showing modeled mineral volumes varying $\mathrm{pH}$ and temperature: $l o w \mathrm{pH}(\mathrm{pH}=$ $2)$, high temperature $\left(40^{\circ} \mathrm{C}\right)$ (top left); slightly acidic $(\mathrm{pH}=5)$, high temperature $\left(40^{\circ} \mathrm{C}\right)$ (top right); low $\mathrm{pH}(\mathrm{pH}=2)$, low temperature $\left(1^{\circ} \mathrm{C}\right)$ (middle left); slightly acidic $(\mathrm{pH}=5)$, low temperature $\left(1^{\circ} \mathrm{C}\right)$ (middle right); low $\mathrm{pH}(\mathrm{pH}=2)$, temperature increasing from $1-40{ }^{\circ} \mathrm{C}$ throughout the column (bottom left); slightly acidic $(\mathrm{pH}=5)$, temperature increasing from 1-40 ${ }^{\circ} \mathrm{C}$ throughout the column (bottom right). All models were run for 1000 years. 


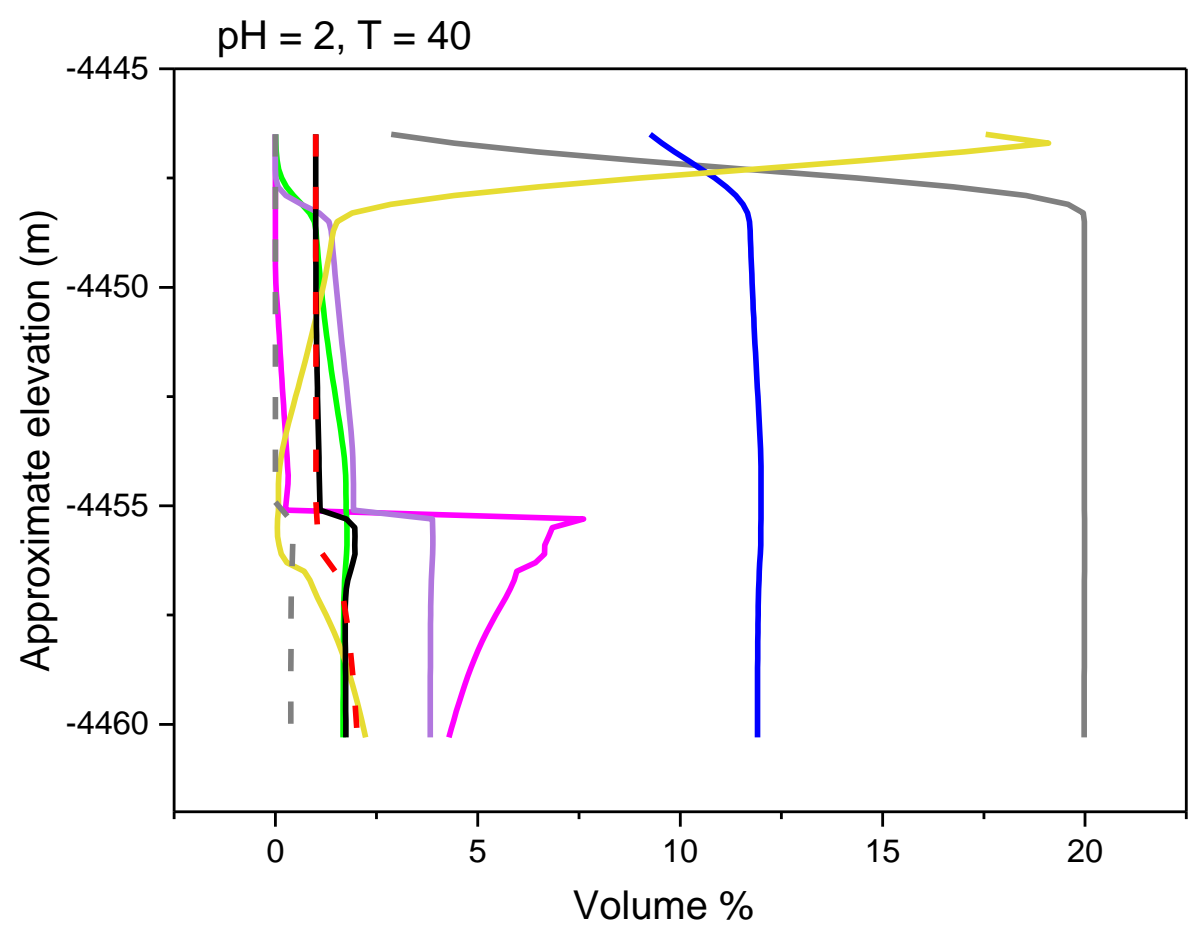

Figure S2. Modeled mineral volumes at $\mathrm{pH}=2$ and $\mathrm{T}=40$. 


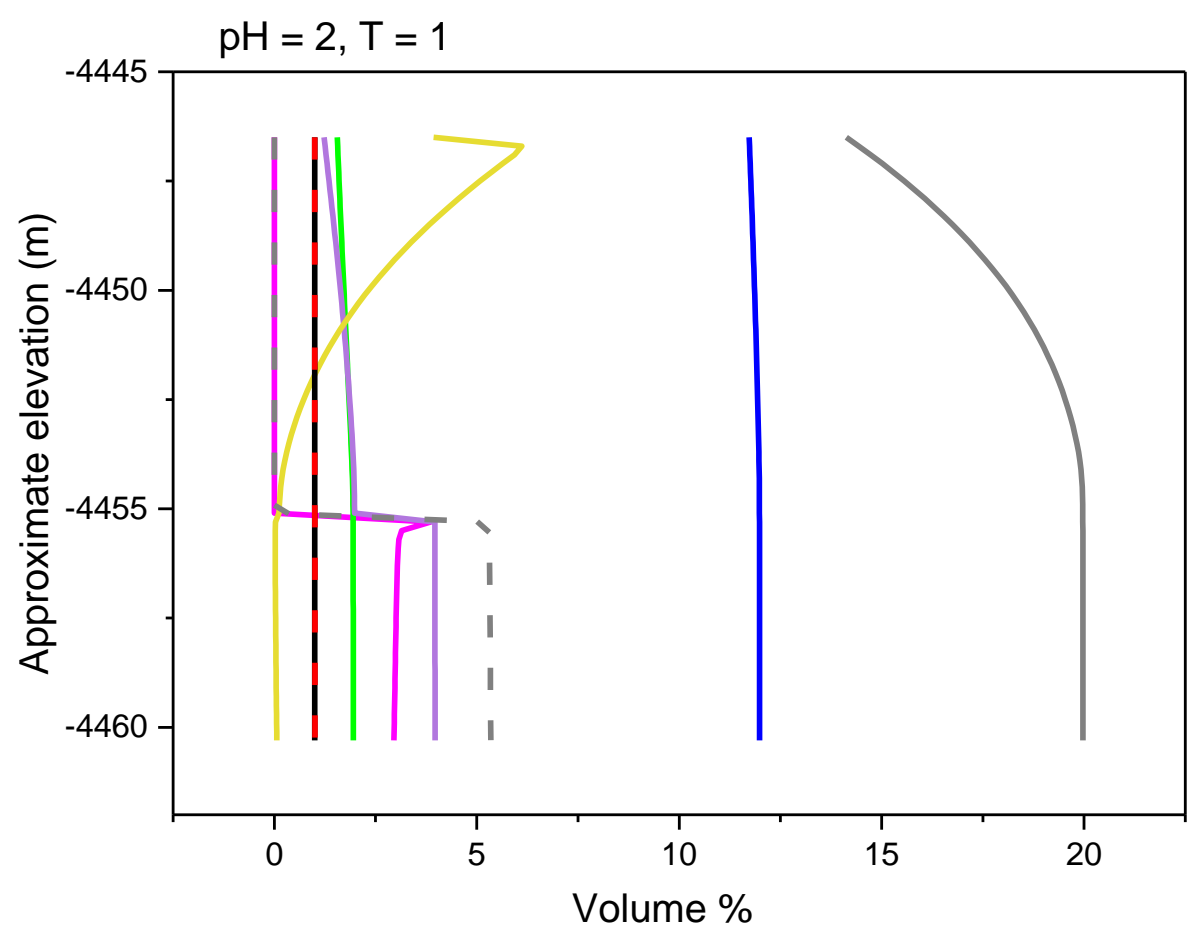

Figure S3. Modeled mineral volumes at $\mathrm{pH}=2$ and $\mathrm{T}=1$. 


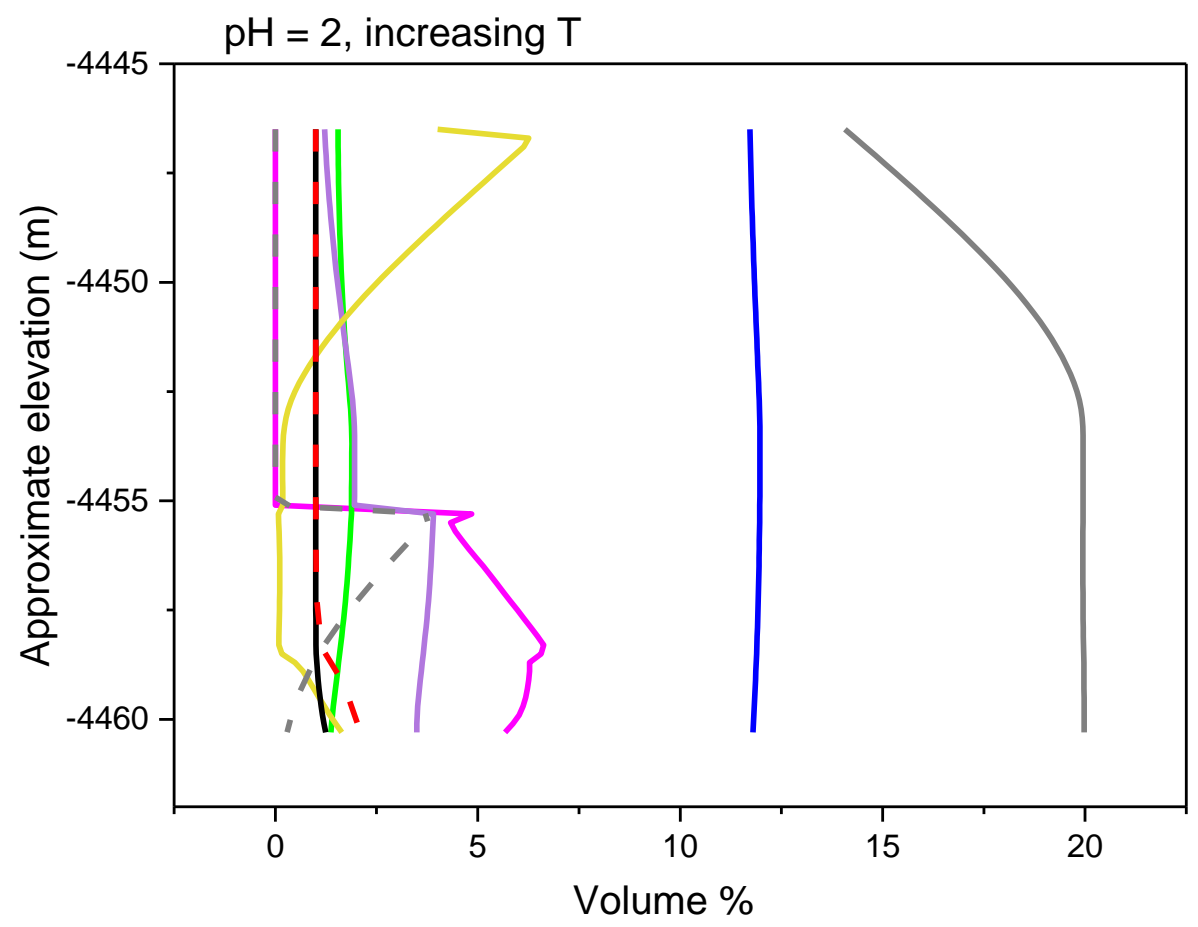

Figure S4. Modeled mineral volumes at $\mathrm{pH}=2$ and increasing $\mathrm{T}$. 


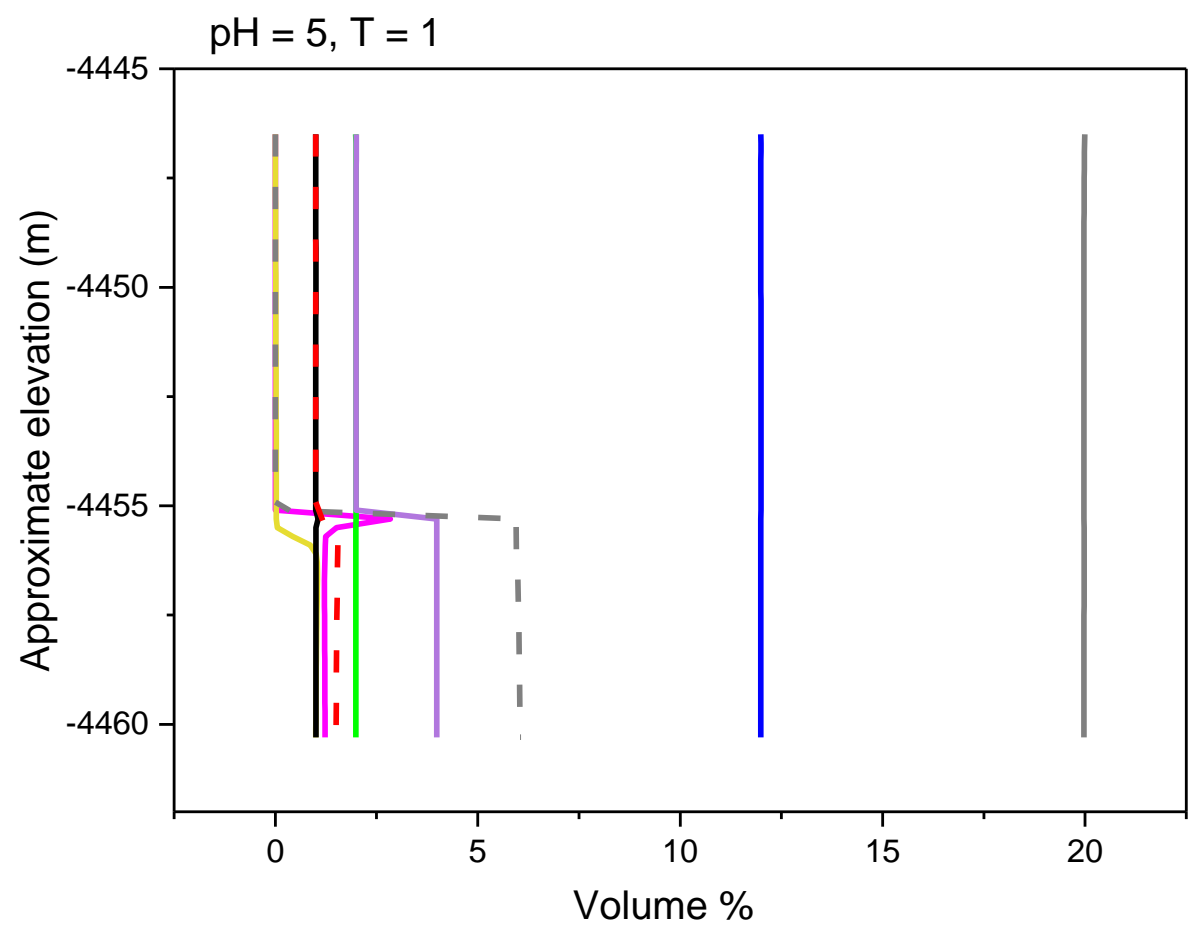

Figure S5. Modeled mineral volumes at $\mathrm{pH}=5$ and $\mathrm{T}=1$. 


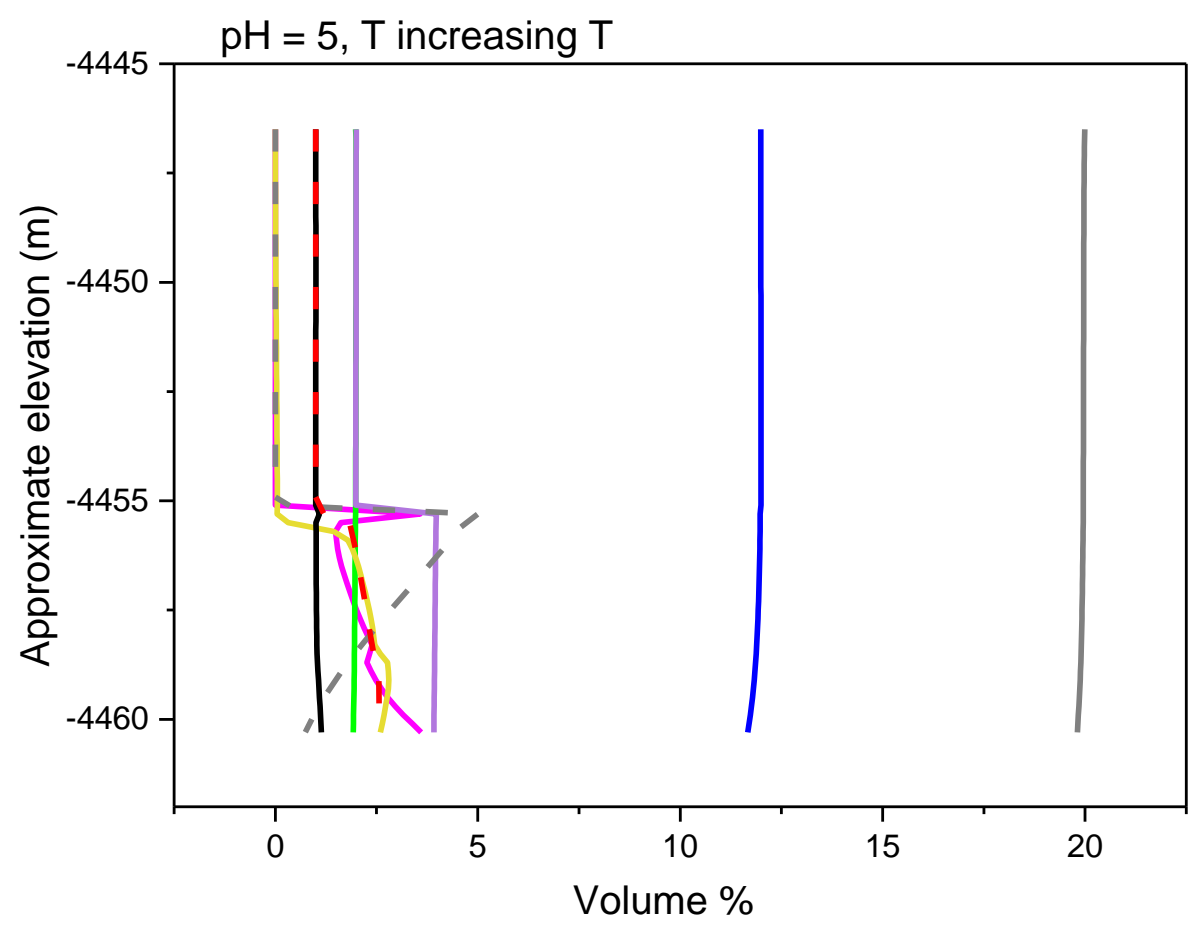

Figure S6. Modeled mineral volumes at $\mathrm{pH}=5$ and increasing $\mathrm{T}$. 


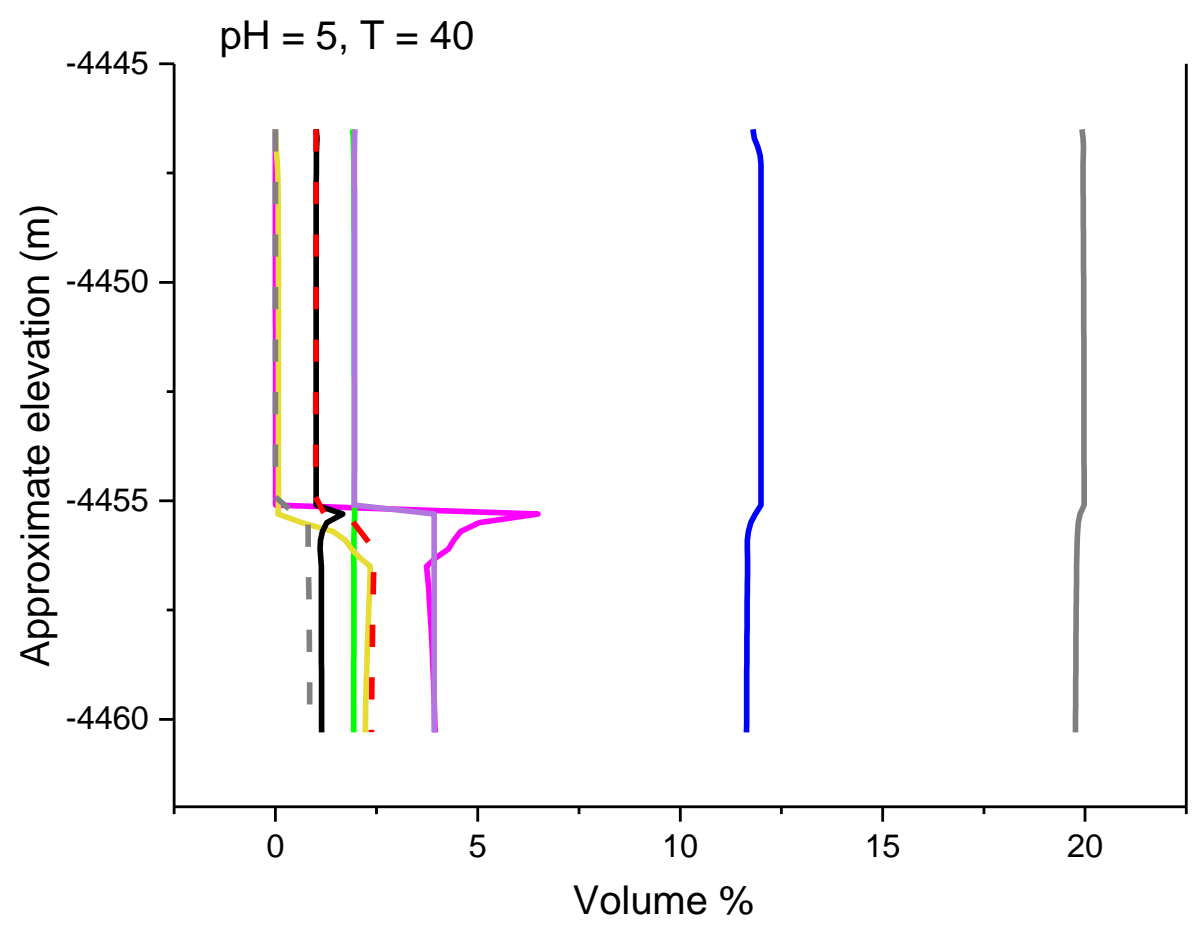

Figure S7. Modeled mineral volumes at $\mathrm{pH}=5$ and $\mathrm{T}=40$. 


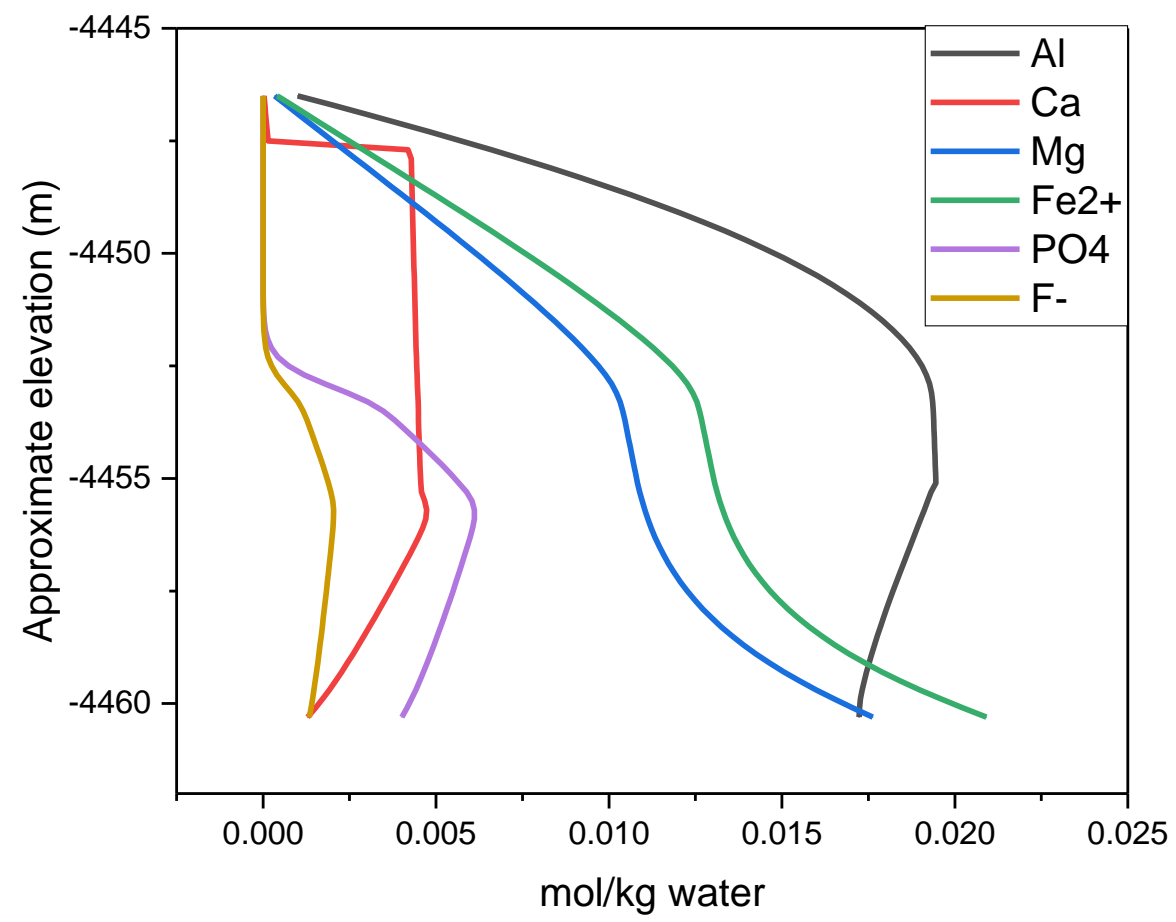

Figure S8. Modeled solution concentrations of $\mathrm{Ca}, \mathrm{Mg}, \mathrm{Fe}^{2+}, \mathrm{PO}_{4}{ }^{3-}$ and $\mathrm{F}^{-}$with depth at $\mathrm{pH}=2$ and $\mathrm{T}=$ increasing. 


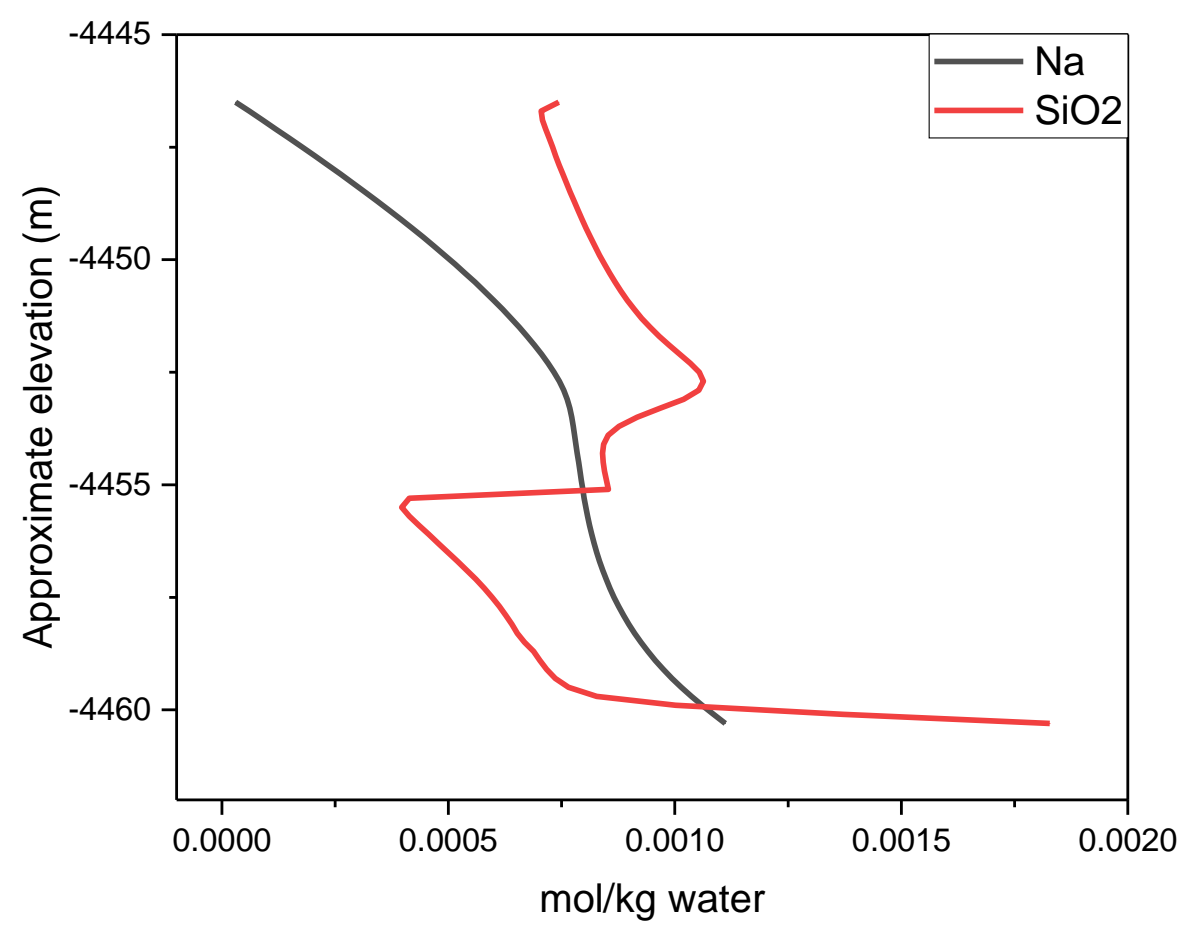

Figure S9. Modeled solution concentrations of $\mathrm{Na}$ and $\mathrm{SiO}_{2}$ with depth at $\mathrm{pH}=2$ and $\mathrm{T}=$ increasing. 


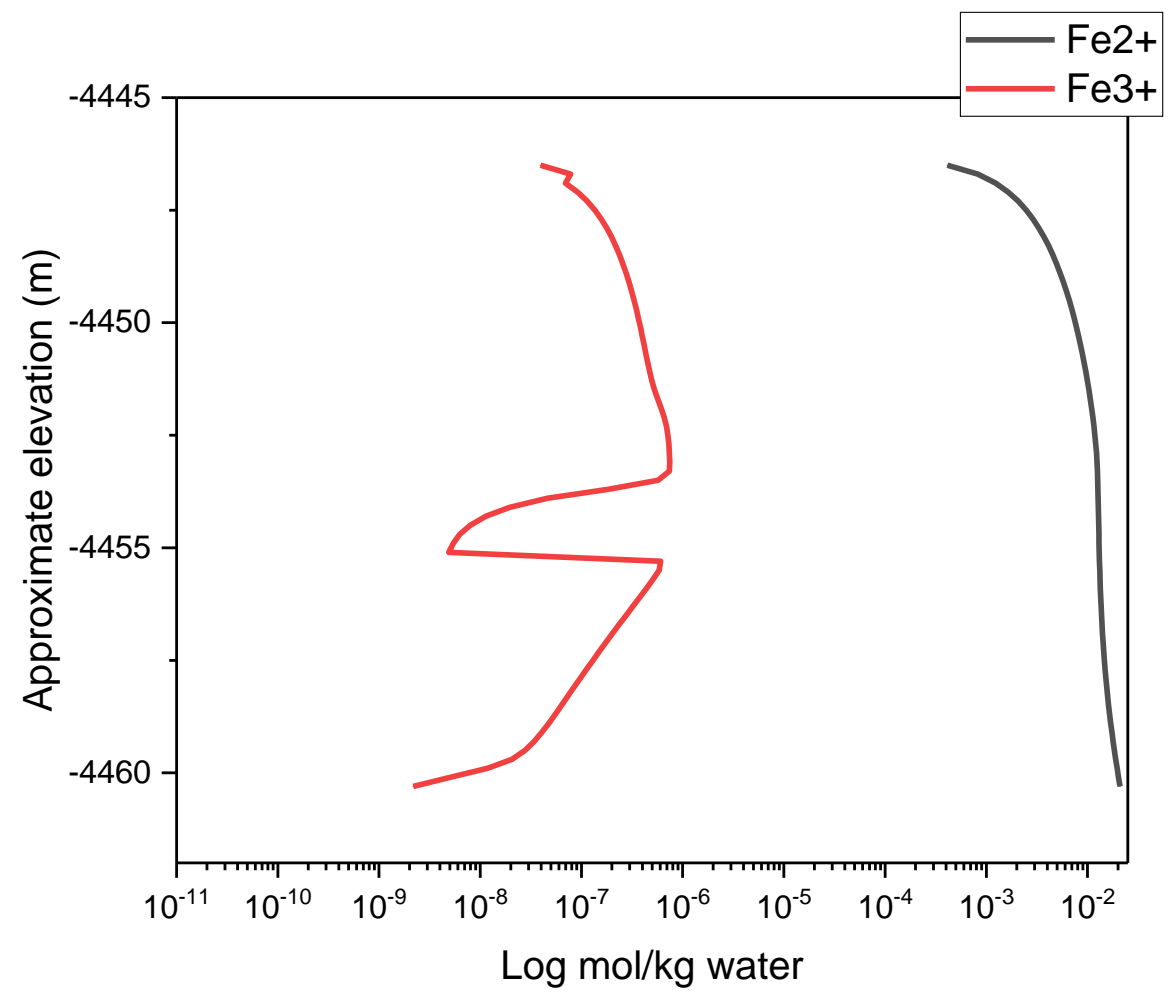

Figure S10. Modeled solution concentrations of $\mathrm{Fe}^{2+}$ and $\mathrm{Fe}^{3+}$ with depth at $\mathrm{pH}=2$ and $\mathrm{T}=$ increasing. 
Table S1. Solution chemistry in $\mathrm{mol} / \mathrm{kg}$ water for the $\mathrm{pH}=2$ increasing temperature condition.

\begin{tabular}{|c|c|c|c|c|c|c|c|c|c|c|c|c|c|c|c|}
\hline Depth & $\mathrm{Al}$ & K & $\mathrm{Na}$ & $\mathrm{Ca}$ & $\mathrm{Mg}$ & $\mathrm{Fe}++$ & $\mathrm{Fe}+++$ & $\mathrm{Ti}$ & $\mathrm{P}$ & $\mathrm{Si}$ & HCO3- & $\mathrm{O} 2(\mathrm{aq})$ & $\mathrm{Cl}-$ & F- & SO42- \\
\hline-4446.5 & $9.97 \mathrm{E}-04$ & $1.00 \mathrm{E}+00$ & $3.02 \mathrm{E}-05$ & $2.47 \mathrm{E}-05$ & 3.32E-04 & 4.12E-04 & $3.95 \mathrm{E}-08$ & $1.00 \mathrm{E}-09$ & $1.00 \mathrm{E}-09$ & 7.44E-04 & $1.00 \mathrm{E}-09$ & $9.87 \mathrm{E}-09$ & $1.00 \mathrm{E}-09$ & $1.00 \mathrm{E}-09$ & $1.00 \mathrm{E}+00$ \\
\hline-4446.7 & $1.98 \mathrm{E}-03$ & $1.00 \mathrm{E}+00$ & $6.02 \mathrm{E}-05$ & 4.93E-05 & 6.66E-04 & $8.24 \mathrm{E}-04$ & $7.81 \mathrm{E}-08$ & $1.00 \mathrm{E}-09$ & $1.00 \mathrm{E}-09$ & $7.05 \mathrm{E}-04$ & $1.00 \mathrm{E}-09$ & $1.95 \mathrm{E}-08$ & $1.00 \mathrm{E}-09$ & $1.00 \mathrm{E}-09$ & $1.00 \mathrm{E}+00$ \\
\hline-4446.9 & $2.94 \mathrm{E}-03$ & $1.00 \mathrm{E}+00$ & 8.99E-05 & $7.36 \mathrm{E}-05$ & $1.00 \mathrm{E}-03$ & $1.24 \mathrm{E}-03$ & $6.96 \mathrm{E}-08$ & $1.00 \mathrm{E}-09$ & $1.00 \mathrm{E}-09$ & 7.08E-04 & $1.00 \mathrm{E}-09$ & 2.89E-08 & 1.00E-09 & $1.00 \mathrm{E}-09$ & $1.00 \mathrm{E}+00$ \\
\hline-4447.1 & $3.89 \mathrm{E}-03$ & $1.00 \mathrm{E}+00$ & 1.19E-04 & 9.77E-05 & $1.34 \mathrm{E}-03$ & $1.65 \mathrm{E}-03$ & $9.20 \mathrm{E}-08$ & $1.00 \mathrm{E}-09$ & $1.00 \mathrm{E}-09$ & $7.15 \mathrm{E}-04$ & $1.00 \mathrm{E}-09$ & $3.81 \mathrm{E}-08$ & $1.00 \mathrm{E}-09$ & $1.00 \mathrm{E}-09$ & $1.00 \mathrm{E}+00$ \\
\hline-4447.3 & $4.82 \mathrm{E}-03$ & $1.00 \mathrm{E}+00$ & 1.49E-04 & $1.22 \mathrm{E}-04$ & $1.67 \mathrm{E}-03$ & $2.07 \mathrm{E}-03$ & $1.14 \mathrm{E}-07$ & $1.00 \mathrm{E}-09$ & $1.00 \mathrm{E}-09$ & 7.23E-04 & $1.00 \mathrm{E}-09$ & $4.70 \mathrm{E}-08$ & 1.00E-09 & $1.00 \mathrm{E}-09$ & $1.00 \mathrm{E}+00$ \\
\hline-4447.5 & $5.72 \mathrm{E}-03$ & $1.00 \mathrm{E}+00$ & 1.77E-04 & $1.45 \mathrm{E}-04$ & $2.01 \mathrm{E}-03$ & $2.48 \mathrm{E}-03$ & $1.36 \mathrm{E}-07$ & 1.00E-09 & $1.00 \mathrm{E}-09$ & 7.30E-04 & $1.00 \mathrm{E}-09$ & $5.56 \mathrm{E}-08$ & 1.00E-09 & $1.00 \mathrm{E}-09$ & $1.00 \mathrm{E}+00$ \\
\hline-4447.7 & $6.60 \mathrm{E}-03$ & $1.00 \mathrm{E}+00$ & 2.06E-04 & 4.19E-03 & 2.34E- 03 & $2.90 \mathrm{E}-03$ & $1.57 \mathrm{E}-07$ & 1.00E-09 & 1.01E-09 & 7.37E-04 & $1.00 \mathrm{E}-09$ & $6.40 \mathrm{E}-08$ & 1.00E-09 & $1.00 \mathrm{E}-09$ & $1.00 \mathrm{E}+00$ \\
\hline-4447.9 & $7.46 \mathrm{E}-03$ & $1.00 \mathrm{E}+00$ & $2.34 \mathrm{E}-04$ & $4.28 \mathrm{E}-03$ & $2.68 \mathrm{E}-03$ & $3.31 \mathrm{E}-03$ & $1.78 \mathrm{E}-07$ & $1.00 \mathrm{E}-09$ & $1.02 \mathrm{E}-09$ & $7.45 \mathrm{E}-04$ & $1.00 \mathrm{E}-09$ & 7.22E-08 & $1.00 \mathrm{E}-09$ & $1.01 \mathrm{E}-09$ & $1.00 \mathrm{E}+00$ \\
\hline-4448.1 & $8.30 \mathrm{E}-03$ & $1.00 \mathrm{E}+00$ & $2.62 \mathrm{E}-04$ & $4.29 \mathrm{E}-03$ & $3.01 \mathrm{E}-03$ & $3.73 \mathrm{E}-03$ & $1.98 \mathrm{E}-07$ & $1.00 \mathrm{E}-09$ & $1.04 \mathrm{E}-09$ & 7.53E-04 & $1.00 \mathrm{E}-09$ & $8.00 \mathrm{E}-08$ & 1.00E-09 & $1.01 \mathrm{E}-09$ & $1.00 \mathrm{E}+00$ \\
\hline-4448.3 & $9.10 \mathrm{E}-03$ & $1.00 \mathrm{E}+00$ & 2.89E-04 & $4.30 \mathrm{E}-03$ & 3.35E-03 & 4.14E-03 & 2.18E-07 & $1.00 \mathrm{E}-09$ & $1.10 \mathrm{E}-09$ & 7.61E-04 & $1.00 \mathrm{E}-09$ & 8.76E-08 & 1.00E-09 & 1.03E-09 & $1.00 \mathrm{E}+00$ \\
\hline-4448.5 & $9.88 \mathrm{E}-03$ & $1.00 \mathrm{E}+00$ & $3.16 \mathrm{E}-04$ & 4.30E-03 & 3.68E-03 & $4.55 \mathrm{E}-03$ & 2.38E-07 & $1.00 \mathrm{E}-09$ & 1.24E-09 & 7.69E-04 & $1.00 \mathrm{E}-09$ & $9.49 \mathrm{E}-08$ & $1.00 \mathrm{E}-09$ & $1.08 \mathrm{E}-09$ & $1.00 \mathrm{E}+00$ \\
\hline-4448.7 & $1.06 \mathrm{E}-02$ & $1.00 \mathrm{E}+00$ & 3.43E-04 & 4.31E-03 & 4.02E-03 & 4.97E-03 & $2.57 \mathrm{E}-07$ & $1.00 \mathrm{E}-09$ & $1.56 \mathrm{E}-09$ & 7.77E-04 & $1.00 \mathrm{E}-09$ & $1.02 \mathrm{E}-07$ & $1.00 \mathrm{E}-09$ & $1.19 \mathrm{E}-09$ & $1.00 \mathrm{E}+00$ \\
\hline-4448.9 & $1.14 \mathrm{E}-02$ & $1.00 \mathrm{E}+00$ & $3.69 \mathrm{E}-04$ & $4.32 \mathrm{E}-03$ & $4.35 \mathrm{E}-03$ & $5.37 \mathrm{E}-03$ & $2.76 \mathrm{E}-07$ & $1.00 \mathrm{E}-09$ & $2.32 \mathrm{E}-09$ & $7.86 \mathrm{E}-04$ & $1.00 \mathrm{E}-09$ & $1.09 \mathrm{E}-07$ & $1.00 \mathrm{E}-09$ & $1.44 \mathrm{E}-09$ & $1.00 \mathrm{E}+00$ \\
\hline-4449.1 & $1.20 \mathrm{E}-02$ & $1.00 \mathrm{E}+00$ & $3.95 \mathrm{E}-04$ & 4.32E-03 & 4.68E-03 & $5.78 \mathrm{E}-03$ & 2.95E-07 & $1.00 \mathrm{E}-09$ & 4.13E-09 & 7.95E-04 & $1.00 \mathrm{E}-09$ & $1.15 \mathrm{E}-07$ & 1.00E-09 & 2.04E-09 & $1.00 \mathrm{E}+00$ \\
\hline-4449.3 & $1.27 \mathrm{E}-02$ & $1.00 \mathrm{E}+00$ & $4.20 \mathrm{E}-04$ & 4.33E-03 & $5.01 \mathrm{E}-03$ & $6.19 \mathrm{E}-03$ & 3.13E-07 & $1.00 \mathrm{E}-09$ & 8.31E-09 & $8.04 \mathrm{E}-04$ & $1.00 \mathrm{E}-09$ & $1.21 \mathrm{E}-07$ & $1.00 \mathrm{E}-09$ & 3.44E-09 & $1.00 \mathrm{E}+00$ \\
\hline-4449.5 & $1.33 \mathrm{E}-02$ & $1.00 \mathrm{E}+00$ & 4.44E-04 & 4.34E-03 & $5.34 \mathrm{E}-03$ & $6.59 \mathrm{E}-03$ & 3.30E-07 & $1.00 \mathrm{E}-09$ & 1.79E-08 & 8.14E-04 & $1.00 \mathrm{E}-09$ & $1.27 \mathrm{E}-07$ & 1.00E-09 & 6.64E-09 & $1.00 \mathrm{E}+00$ \\
\hline-4449.7 & $1.39 \mathrm{E}-02$ & $1.00 \mathrm{E}+00$ & $4.68 \mathrm{E}-04$ & $4.34 \mathrm{E}-03$ & $5.67 \mathrm{E}-03$ & $6.99 \mathrm{E}-03$ & $3.48 \mathrm{E}-07$ & $1.00 \mathrm{E}-09$ & $3.96 \mathrm{E}-08$ & $8.24 \mathrm{E}-04$ & $1.00 \mathrm{E}-09$ & $1.33 \mathrm{E}-07$ & $1.00 \mathrm{E}-09$ & $1.39 \mathrm{E}-08$ & $1.00 \mathrm{E}+00$ \\
\hline-4449.9 & $1.45 \mathrm{E}-02$ & $1.00 \mathrm{E}+00$ & 4.92E-04 & 4.35E-03 & $5.99 \mathrm{E}-03$ & $7.38 \mathrm{E}-03$ & $3.65 \mathrm{E}-07$ & $1.00 \mathrm{E}-09$ & 8.81E-08 & 8.34E-04 & $1.00 \mathrm{E}-09$ & $1.38 \mathrm{E}-07$ & $1.00 \mathrm{E}-09$ & $3.00 \mathrm{E}-08$ & $1.00 \mathrm{E}+00$ \\
\hline-4450.1 & $1.50 \mathrm{E}-02$ & $1.00 \mathrm{E}+00$ & $5.15 \mathrm{E}-04$ & $4.36 \mathrm{E}-03$ & $6.31 \mathrm{E}-03$ & $7.77 \mathrm{E}-03$ & $3.82 \mathrm{E}-07$ & $1.00 \mathrm{E}-09$ & $1.95 \mathrm{E}-07$ & $8.45 \mathrm{E}-04$ & $1.00 \mathrm{E}-09$ & $1.43 \mathrm{E}-07$ & $1.00 \mathrm{E}-09$ & $6.55 \mathrm{E}-08$ & $1.00 \mathrm{E}+00$ \\
\hline-4450.3 & $1.56 \mathrm{E}-02$ & $1.00 \mathrm{E}+00$ & $5.37 \mathrm{E}-04$ & $4.36 \mathrm{E}-03$ & $6.62 \mathrm{E}-03$ & $8.16 \mathrm{E}-03$ & $3.99 \mathrm{E}-07$ & $1.00 \mathrm{E}-09$ & 4.26E-07 & $8.56 \mathrm{E}-04$ & $1.00 \mathrm{E}-09$ & $1.48 \mathrm{E}-07$ & $1.00 \mathrm{E}-09$ & $1.43 \mathrm{E}-07$ & $1.00 \mathrm{E}+00$ \\
\hline-4450.5 & $1.60 \mathrm{E}-02$ & $1.00 \mathrm{E}+00$ & $5.59 \mathrm{E}-04$ & 4.37E-03 & $6.94 \mathrm{E}-03$ & $8.54 \mathrm{E}-03$ & $4.16 \mathrm{E}-07$ & $1.00 \mathrm{E}-09$ & $9.19 \mathrm{E}-07$ & 8.68E-04 & $1.00 \mathrm{E}-09$ & $1.53 \mathrm{E}-07$ & $1.00 \mathrm{E}-09$ & $3.07 \mathrm{E}-07$ & $1.00 \mathrm{E}+00$ \\
\hline-4450.7 & $1.65 \mathrm{E}-02$ & $1.00 \mathrm{E}+00$ & $5.80 \mathrm{E}-04$ & $4.38 \mathrm{E}-03$ & $7.24 \mathrm{E}-03$ & $8.91 \mathrm{E}-03$ & $4.34 \mathrm{E}-07$ & $1.00 \mathrm{E}-09$ & $1.96 \mathrm{E}-06$ & 8.81E-04 & 1.00E-09 & $1.57 \mathrm{E}-07$ & $1.00 \mathrm{E}-09$ & $6.53 \mathrm{E}-07$ & $1.00 \mathrm{E}+00$ \\
\hline-4450.9 & $1.69 \mathrm{E}-02$ & $1.00 \mathrm{E}+00$ & $6.00 \mathrm{E}-04$ & $4.38 \mathrm{E}-03$ & $7.55 \mathrm{E}-03$ & $9.28 \mathrm{E}-03$ & $4.52 \mathrm{E}-07$ & $1.00 \mathrm{E}-09$ & 4.11E-06 & $8.95 \mathrm{E}-04$ & $1.00 \mathrm{E}-09$ & $1.61 \mathrm{E}-07$ & $1.00 \mathrm{E}-09$ & $1.37 \mathrm{E}-06$ & $1.00 \mathrm{E}+00$ \\
\hline-4451.1 & $1.73 \mathrm{E}-02$ & $1.00 \mathrm{E}+00$ & $6.19 \mathrm{E}-04$ & 4.39E-03 & $7.84 \mathrm{E}-03$ & $9.63 \mathrm{E}-03$ & $4.73 \mathrm{E}-07$ & $1.00 \mathrm{E}-09$ & $8.48 \mathrm{E}-06$ & $9.10 \mathrm{E}-04$ & $1.00 \mathrm{E}-09$ & $1.65 \mathrm{E}-07$ & $1.00 \mathrm{E}-09$ & $2.83 \mathrm{E}-06$ & $1.00 \mathrm{E}+00$ \\
\hline-4451.3 & $1.76 \mathrm{E}-02$ & $1.00 \mathrm{E}+00$ & $6.38 \mathrm{E}-04$ & $4.40 \mathrm{E}-03$ & $8.14 \mathrm{E}-03$ & $9.99 \mathrm{E}-03$ & $4.97 \mathrm{E}-07$ & $1.00 \mathrm{E}-09$ & $1.72 \mathrm{E}-05$ & $9.27 \mathrm{E}-04$ & $1.00 \mathrm{E}-09$ & $1.68 \mathrm{E}-07$ & $1.00 \mathrm{E}-09$ & $5.74 \mathrm{E}-06$ & $1.00 \mathrm{E}+00$ \\
\hline-4451.5 & $1.79 \mathrm{E}-02$ & $1.00 \mathrm{E}+00$ & $6.56 \mathrm{E}-04$ & $4.40 \mathrm{E}-03$ & $8.42 \mathrm{E}-03$ & $1.03 \mathrm{E}-02$ & $5.28 \mathrm{E}-07$ & $1.00 \mathrm{E}-09$ & $3.43 \mathrm{E}-05$ & $9.45 \mathrm{E}-04$ & 1.00E-09 & $1.71 \mathrm{E}-07$ & $1.00 \mathrm{E}-09$ & $1.14 \mathrm{E}-05$ & $1.00 \mathrm{E}+00$ \\
\hline-4451.7 & $1.82 \mathrm{E}-02$ & $1.00 \mathrm{E}+00$ & $6.74 \mathrm{E}-04$ & 4.41E-03 & $8.70 \mathrm{E}-03$ & $1.07 \mathrm{E}-02$ & $5.67 \mathrm{E}-07$ & $1.00 \mathrm{E}-09$ & $6.70 \mathrm{E}-05$ & $9.65 \mathrm{E}-04$ & $1.00 \mathrm{E}-09$ & $1.74 \mathrm{E}-07$ & $1.00 \mathrm{E}-09$ & 2.23E-05 & $1.00 \mathrm{E}+00$ \\
\hline-4451.9 & $1.85 \mathrm{E}-02$ & $1.00 \mathrm{E}+00$ & $6.90 \mathrm{E}-04$ & 4.42E- 03 & 8.97E-03 & $1.10 \mathrm{E}-02$ & 6.13E-07 & 1.00E-09 & $1.28 \mathrm{E}-04$ & $9.87 \mathrm{E}-04$ & $1.00 \mathrm{E}-09$ & $1.77 \mathrm{E}-07$ & $1.00 \mathrm{E}-09$ & $4.27 \mathrm{E}-05$ & $1.00 \mathrm{E}+00$ \\
\hline-4452.1 & $1.87 \mathrm{E}-02$ & $1.00 \mathrm{E}+00$ & 7.06E-04 & $4.42 \mathrm{E}-03$ & $9.22 \mathrm{E}-03$ & $1.13 \mathrm{E}-02$ & $6.56 \mathrm{E}-07$ & $1.00 \mathrm{E}-09$ & $2.38 \mathrm{E}-04$ & $1.01 \mathrm{E}-03$ & $1.00 \mathrm{E}-09$ & $1.79 \mathrm{E}-07$ & $1.00 \mathrm{E}-09$ & 7.93E-05 & $1.00 \mathrm{E}+00$ \\
\hline-4452.3 & $1.89 \mathrm{E}-02$ & $1.00 \mathrm{E}+00$ & $7.20 \mathrm{E}-04$ & 4.43E-03 & 9.47E-03 & $1.16 \mathrm{E}-02$ & $6.91 \mathrm{E}-07$ & $1.00 \mathrm{E}-09$ & 4.28E-04 & $1.03 \mathrm{E}-03$ & 1.00E-09 & $1.81 \mathrm{E}-07$ & $1.00 \mathrm{E}-09$ & $1.43 \mathrm{E}-04$ & $1.00 \mathrm{E}+00$ \\
\hline-4452.5 & $1.91 \mathrm{E}-02$ & $1.00 \mathrm{E}+00$ & 7.33E-04 & 4.44E-03 & $9.69 \mathrm{E}-03$ & $1.18 \mathrm{E}-02$ & $7.15 \mathrm{E}-07$ & $1.00 \mathrm{E}-09$ & 7.35E-04 & $1.05 \mathrm{E}-03$ & 1.00E-09 & $1.83 \mathrm{E}-07$ & $1.00 \mathrm{E}-09$ & $2.45 \mathrm{E}-04$ & $1.00 \mathrm{E}+00$ \\
\hline-4452.7 & $1.92 \mathrm{E}-02$ & $1.00 \mathrm{E}+00$ & 7.45E-04 & 4.45E-03 & $9.90 \mathrm{E}-03$ & $1.21 \mathrm{E}-02$ & 7.30E-07 & $1.00 \mathrm{E}-09$ & $1.19 \mathrm{E}-03$ & $1.06 \mathrm{E}-03$ & $1.00 \mathrm{E}-09$ & $1.85 \mathrm{E}-07$ & $1.00 \mathrm{E}-09$ & 3.97E-04 & $1.00 \mathrm{E}+00$ \\
\hline-4452.9 & $1.93 \mathrm{E}-02$ & $1.00 \mathrm{E}+00$ & $7.55 \mathrm{E}-04$ & 4.46E-03 & $1.01 \mathrm{E}-02$ & $1.23 \mathrm{E}-02$ & 7.40E-07 & $1.00 \mathrm{E}-09$ & $1.79 \mathrm{E}-03$ & $1.05 \mathrm{E}-03$ & $1.00 \mathrm{E}-09$ & $1.86 \mathrm{E}-07$ & 1.00E-09 & 5.97E-04 & $1.00 \mathrm{E}+00$ \\
\hline-4453.1 & $1.93 \mathrm{E}-02$ & $1.00 \mathrm{E}+00$ & 7.63E-04 & 4.47E-03 & $1.02 \mathrm{E}-02$ & $1.24 \mathrm{E}-02$ & 7.45E-07 & $1.00 \mathrm{E}-09$ & $2.45 \mathrm{E}-03$ & $1.02 \mathrm{E}-03$ & $1.00 \mathrm{E}-09$ & $1.87 \mathrm{E}-07$ & 1.00E-09 & 8.16E-04 & $1.00 \mathrm{E}+00$ \\
\hline-4453.3 & $1.94 \mathrm{E}-02$ & $1.00 \mathrm{E}+00$ & $7.68 \mathrm{E}-04$ & $4.48 \mathrm{E}-03$ & $1.03 \mathrm{E}-02$ & $1.25 \mathrm{E}-02$ & $7.38 \mathrm{E}-07$ & $1.00 \mathrm{E}-09$ & 3.03E-03 & $9.68 \mathrm{E}-04$ & $1.00 \mathrm{E}-09$ & $1.85 \mathrm{E}-07$ & $1.00 \mathrm{E}-09$ & $1.01 \mathrm{E}-03$ & $9.99 \mathrm{E}-01$ \\
\hline-4453.5 & $1.94 \mathrm{E}-02$ & $1.00 \mathrm{E}+00$ & 7.72E-04 & 4.49E-03 & $1.04 \mathrm{E}-02$ & $1.26 \mathrm{E}-02$ & 5.71E-07 & $1.00 \mathrm{E}-09$ & 3.47E-03 & $9.16 \mathrm{E}-04$ & $1.00 \mathrm{E}-09$ & 1.43E-07 & 1.00E-09 & $1.16 \mathrm{E}-03$ & $9.98 \mathrm{E}-01$ \\
\hline-4453.7 & $1.94 \mathrm{E}-02$ & $1.00 \mathrm{E}+00$ & 7.76E-04 & 4.49E-03 & $1.05 \mathrm{E}-02$ & $1.27 \mathrm{E}-02$ & $1.83 \mathrm{E}-07$ & $1.00 \mathrm{E}-09$ & $3.80 \mathrm{E}-03$ & 8.77E-04 & $1.00 \mathrm{E}-09$ & $4.57 \mathrm{E}-08$ & $1.00 \mathrm{E}-09$ & $1.27 \mathrm{E}-03$ & $9.98 \mathrm{E}-01$ \\
\hline-4453.9 & $1.94 \mathrm{E}-02$ & $1.00 \mathrm{E}+00$ & 7.79E-04 & 4.50E-03 & $1.05 \mathrm{E}-02$ & $1.27 \mathrm{E}-02$ & 4.61E-08 & $1.00 \mathrm{E}-09$ & $4.08 \mathrm{E}-03$ & 8.53E-04 & $1.00 \mathrm{E}-09$ & 1.15E-08 & $1.00 \mathrm{E}-09$ & $1.36 \mathrm{E}-03$ & $9.97 \mathrm{E}-01$ \\
\hline-4454.1 & $1.94 \mathrm{E}-02$ & $1.00 \mathrm{E}+00$ & 7.82E-04 & $4.51 \mathrm{E}-03$ & $1.06 \mathrm{E}-02$ & $1.28 \mathrm{E}-02$ & $1.96 \mathrm{E}-08$ & 1.00E-09 & 4.36E-03 & 8.43E-04 & $1.00 \mathrm{E}-09$ & $4.90 \mathrm{E}-09$ & $1.00 \mathrm{E}-09$ & $1.45 \mathrm{E}-03$ & $9.97 \mathrm{E}-01$ \\
\hline-4454.3 & $1.94 \mathrm{E}-02$ & $1.00 \mathrm{E}+00$ & 7.84E-04 & $4.52 \mathrm{E}-03$ & $1.06 \mathrm{E}-02$ & $1.28 \mathrm{E}-02$ & $1.14 \mathrm{E}-08$ & $1.00 \mathrm{E}-09$ & $4.64 \mathrm{E}-03$ & $8.40 \mathrm{E}-04$ & $1.00 \mathrm{E}-09$ & $2.84 \mathrm{E}-09$ & $1.00 \mathrm{E}-09$ & $1.55 \mathrm{E}-03$ & $9.96 \mathrm{E}-01$ \\
\hline
\end{tabular}




\begin{tabular}{|c|c|c|c|c|c|c|c|c|c|c|c|c|c|c|c|}
\hline-4454.5 & $1.94 \mathrm{E}-02$ & $1.00 \mathrm{E}+00$ & 7.87E-04 & $4.53 \mathrm{E}-03$ & $1.07 \mathrm{E}-02$ & $1.29 \mathrm{E}-02$ & 7.96E-09 & $1.00 \mathrm{E}-09$ & $4.91 \mathrm{E}-03$ & $8.42 \mathrm{E}-04$ & $1.00 \mathrm{E}-09$ & $1.99 \mathrm{E}-09$ & $1.00 \mathrm{E}-09$ & $1.64 \mathrm{E}-03$ & $9.96 \mathrm{E}-01$ \\
\hline-4454.7 & $1.94 \mathrm{E}-02$ & $1.00 \mathrm{E}+00$ & $7.90 \mathrm{E}-04$ & $4.54 \mathrm{E}-03$ & $1.07 \mathrm{E}-02$ & $1.29 \mathrm{E}-02$ & $6.30 \mathrm{E}-09$ & $1.00 \mathrm{E}-09$ & $5.17 \mathrm{E}-03$ & $8.45 \mathrm{E}-04$ & $1.00 \mathrm{E}-09$ & $1.58 \mathrm{E}-09$ & $1.00 \mathrm{E}-09$ & $1.72 \mathrm{E}-03$ & $9.95 \mathrm{E}-01$ \\
\hline-4454.9 & $1.94 \mathrm{E}-02$ & $1.00 \mathrm{E}+00$ & 7.94E-04 & $4.55 \mathrm{E}-03$ & $1.08 \mathrm{E}-02$ & $1.30 \mathrm{E}-02$ & $5.41 \mathrm{E}-09$ & $1.00 \mathrm{E}-09$ & $5.43 \mathrm{E}-03$ & 8.49E-04 & $1.00 \mathrm{E}-09$ & $1.35 \mathrm{E}-09$ & $1.00 \mathrm{E}-09$ & $1.81 \mathrm{E}-03$ & $9.95 \mathrm{E}-01$ \\
\hline-4455.1 & $1.95 \mathrm{E}-02$ & $1.00 \mathrm{E}+00$ & 7.97E-04 & $4.56 \mathrm{E}-03$ & $1.08 \mathrm{E}-02$ & $1.31 \mathrm{E}-02$ & $4.90 \mathrm{E}-09$ & $1.00 \mathrm{E}-09$ & $5.66 \mathrm{E}-03$ & $8.53 \mathrm{E}-04$ & $1.00 \mathrm{E}-09$ & $1.22 \mathrm{E}-09$ & $1.00 \mathrm{E}-09$ & $1.89 \mathrm{E}-03$ & $9.95 \mathrm{E}-01$ \\
\hline-4455.3 & $1.93 \mathrm{E}-02$ & $1.00 \mathrm{E}+00$ & 8.00E-04 & $4.58 \mathrm{E}-03$ & $1.09 \mathrm{E}-02$ & $1.31 \mathrm{E}-02$ & 6.07E-07 & 1.00E-09 & $5.88 \mathrm{E}-03$ & 4.14E-04 & $1.00 \mathrm{E}-09$ & $1.52 \mathrm{E}-07$ & 1.00E-09 & $1.96 \mathrm{E}-03$ & $9.94 \mathrm{E}-01$ \\
\hline-4455.5 & $1.92 \mathrm{E}-02$ & $1.00 \mathrm{E}+00$ & 8.03E-04 & 4.67E-03 & $1.10 \mathrm{E}-02$ & $1.32 \mathrm{E}-02$ & $5.86 \mathrm{E}-07$ & $1.00 \mathrm{E}-09$ & $6.04 \mathrm{E}-03$ & $3.97 \mathrm{E}-04$ & $1.00 \mathrm{E}-09$ & $1.46 \mathrm{E}-07$ & $1.00 \mathrm{E}-09$ & $2.01 \mathrm{E}-03$ & $9.94 \mathrm{E}-01$ \\
\hline-4455.7 & $1.91 \mathrm{E}-02$ & $1.00 \mathrm{E}+00$ & 8.07E-04 & 4.73E-03 & $1.10 \mathrm{E}-02$ & 1.33E-02 & $5.11 \mathrm{E}-07$ & 1.00E-09 & 6.11E-03 & 4.14E-04 & $1.00 \mathrm{E}-09$ & $1.28 \mathrm{E}-07$ & 1.00E-09 & $2.04 \mathrm{E}-03$ & $9.94 \mathrm{E}-01$ \\
\hline-4455.9 & $1.90 \mathrm{E}-02$ & $1.00 \mathrm{E}+00$ & 8.12E-04 & 4.70E-03 & $1.11 \mathrm{E}-02$ & $1.34 \mathrm{E}-02$ & $4.40 \mathrm{E}-07$ & 1.00E-09 & 6.11E-03 & 4.35E-04 & $1.00 \mathrm{E}-09$ & $1.10 \mathrm{E}-07$ & $1.00 \mathrm{E}-09$ & $2.04 \mathrm{E}-03$ & $9.94 \mathrm{E}-01$ \\
\hline-4456.1 & $1.89 \mathrm{E}-02$ & $1.00 \mathrm{E}+00$ & $8.16 \mathrm{E}-04$ & $4.62 \mathrm{E}-03$ & $1.12 \mathrm{E}-02$ & $1.35 \mathrm{E}-02$ & 3.77E-07 & $1.00 \mathrm{E}-09$ & $6.06 \mathrm{E}-03$ & $4.56 \mathrm{E}-04$ & $1.00 \mathrm{E}-09$ & $9.42 \mathrm{E}-08$ & $1.00 \mathrm{E}-09$ & 2.02E-03 & $9.93 \mathrm{E}-01$ \\
\hline-4456.3 & $1.88 \mathrm{E}-02$ & $1.00 \mathrm{E}+00$ & 8.22E-04 & $4.50 \mathrm{E}-03$ & 1.13E-02 & $1.36 \mathrm{E}-02$ & 3.22E-07 & 1.00E-09 & 5.99E-03 & 4.78E-04 & $1.00 \mathrm{E}-09$ & 8.06E-08 & 1.00E-09 & $2.00 \mathrm{E}-03$ & $9.93 \mathrm{E}-01$ \\
\hline-4456.5 & $1.87 \mathrm{E}-02$ & $1.00 \mathrm{E}+00$ & 8.27E-04 & 4.37E-03 & $1.14 \mathrm{E}-02$ & $1.37 \mathrm{E}-02$ & 2.76E-07 & 1.00E-09 & 5.91E-03 & 4.99E-04 & $1.00 \mathrm{E}-09$ & $6.90 \mathrm{E}-08$ & 1.00E-09 & $1.97 \mathrm{E}-03$ & $9.93 \mathrm{E}-01$ \\
\hline-4456.7 & $1.86 \mathrm{E}-02$ & $1.00 \mathrm{E}+00$ & 8.34E-04 & $4.23 \mathrm{E}-03$ & $1.16 \mathrm{E}-02$ & $1.39 \mathrm{E}-02$ & 2.37E-07 & 1.00E-09 & $5.83 \mathrm{E}-03$ & $5.20 \mathrm{E}-04$ & $1.00 \mathrm{E}-09$ & 5.91E-08 & 1.00E-09 & $1.94 \mathrm{E}-03$ & $9.93 \mathrm{E}-01$ \\
\hline-4456.9 & $1.85 \mathrm{E}-02$ & $1.00 \mathrm{E}+00$ & 8.41E-04 & $4.09 \mathrm{E}-03$ & $1.17 \mathrm{E}-02$ & $1.41 \mathrm{E}-02$ & 2.03E-07 & 1.00E-09 & $5.75 \mathrm{E}-03$ & 5.41E-04 & 1.00E-09 & $5.07 \mathrm{E}-08$ & 1.00E-09 & $1.92 \mathrm{E}-03$ & $9.93 \mathrm{E}-01$ \\
\hline-4457.1 & $1.84 \mathrm{E}-02$ & $1.00 \mathrm{E}+00$ & 8.48E-04 & 3.95E-03 & 1.19E-02 & $1.42 \mathrm{E}-02$ & $1.75 \mathrm{E}-07$ & 1.00E-09 & $5.66 \mathrm{E}-03$ & 5.61E-04 & $1.00 \mathrm{E}-09$ & 4.36E-08 & 1.00E-09 & $1.89 \mathrm{E}-03$ & $9.93 \mathrm{E}-01$ \\
\hline-4457.3 & $1.83 \mathrm{E}-02$ & $1.00 \mathrm{E}+00$ & 8.57E-04 & $3.81 \mathrm{E}-03$ & $1.21 \mathrm{E}-02$ & $1.44 \mathrm{E}-02$ & $1.50 \mathrm{E}-07$ & $1.00 \mathrm{E}-09$ & $5.58 \mathrm{E}-03$ & $5.79 \mathrm{E}-04$ & $1.00 \mathrm{E}-09$ & $3.76 \mathrm{E}-08$ & $1.00 \mathrm{E}-09$ & $1.86 \mathrm{E}-03$ & $9.93 \mathrm{E}-01$ \\
\hline-4457.5 & $1.82 \mathrm{E}-02$ & $1.00 \mathrm{E}+00$ & $8.66 \mathrm{E}-04$ & $3.66 \mathrm{E}-03$ & $1.23 \mathrm{E}-02$ & $1.47 \mathrm{E}-02$ & $1.30 \mathrm{E}-07$ & $1.00 \mathrm{E}-09$ & $5.49 \mathrm{E}-03$ & $5.97 \mathrm{E}-04$ & $1.00 \mathrm{E}-09$ & 3.25E-08 & 1.00E-09 & $1.83 \mathrm{E}-03$ & $9.93 \mathrm{E}-01$ \\
\hline-4457.7 & $1.81 \mathrm{E}-02$ & $1.00 \mathrm{E}+00$ & 8.76E-04 & $3.52 \mathrm{E}-03$ & $1.25 \mathrm{E}-02$ & 1.49E-02 & $1.12 \mathrm{E}-07$ & 1.00E-09 & $5.40 \mathrm{E}-03$ & 6.13E-04 & 1.00E-09 & $2.81 \mathrm{E}-08$ & 1.00E-09 & $1.80 \mathrm{E}-03$ & $9.93 \mathrm{E}-01$ \\
\hline-4457.9 & $1.80 \mathrm{E}-02$ & $1.00 \mathrm{E}+00$ & 8.87E-04 & $3.37 \mathrm{E}-03$ & $1.27 \mathrm{E}-02$ & $1.52 \mathrm{E}-02$ & $9.73 \mathrm{E}-08$ & $1.00 \mathrm{E}-09$ & $5.32 \mathrm{E}-03$ & $6.27 \mathrm{E}-04$ & $1.00 \mathrm{E}-09$ & $2.43 \mathrm{E}-08$ & $1.00 \mathrm{E}-09$ & $1.77 \mathrm{E}-03$ & $9.93 \mathrm{E}-01$ \\
\hline-4458.1 & $1.79 \mathrm{E}-02$ & $1.00 \mathrm{E}+00$ & $8.98 \mathrm{E}-04$ & $3.22 \mathrm{E}-03$ & $1.30 \mathrm{E}-02$ & $1.55 \mathrm{E}-02$ & $8.43 \mathrm{E}-08$ & $1.00 \mathrm{E}-09$ & $5.22 \mathrm{E}-03$ & $6.40 \mathrm{E}-04$ & $1.00 \mathrm{E}-09$ & $2.11 \mathrm{E}-08$ & $1.00 \mathrm{E}-09$ & $1.74 \mathrm{E}-03$ & $9.94 \mathrm{E}-01$ \\
\hline-4458.3 & $1.78 \mathrm{E}-02$ & $1.00 \mathrm{E}+00$ & $9.11 \mathrm{E}-04$ & $3.07 \mathrm{E}-03$ & $1.33 \mathrm{E}-02$ & $1.58 \mathrm{E}-02$ & 7.32E-08 & $1.00 \mathrm{E}-09$ & $5.13 \mathrm{E}-03$ & $6.52 \mathrm{E}-04$ & $1.00 \mathrm{E}-09$ & $1.83 \mathrm{E}-08$ & $1.00 \mathrm{E}-09$ & $1.71 \mathrm{E}-03$ & $9.94 \mathrm{E}-01$ \\
\hline-4458.5 & $1.78 \mathrm{E}-02$ & $1.00 \mathrm{E}+00$ & $9.25 \mathrm{E}-04$ & $2.92 \mathrm{E}-03$ & $1.36 \mathrm{E}-02$ & $1.61 \mathrm{E}-02$ & $6.34 \mathrm{E}-08$ & $1.00 \mathrm{E}-09$ & $5.04 \mathrm{E}-03$ & $6.68 \mathrm{E}-04$ & $1.00 \mathrm{E}-09$ & $1.59 \mathrm{E}-08$ & $1.00 \mathrm{E}-09$ & $1.68 \mathrm{E}-03$ & $9.94 \mathrm{E}-01$ \\
\hline-4458.7 & $1.77 \mathrm{E}-02$ & $1.00 \mathrm{E}+00$ & $9.40 \mathrm{E}-04$ & $2.76 \mathrm{E}-03$ & $1.39 \mathrm{E}-02$ & $1.65 \mathrm{E}-02$ & $5.51 \mathrm{E}-08$ & $1.00 \mathrm{E}-09$ & $4.94 \mathrm{E}-03$ & $6.88 \mathrm{E}-04$ & $1.00 \mathrm{E}-09$ & $1.38 \mathrm{E}-08$ & $1.00 \mathrm{E}-09$ & $1.65 \mathrm{E}-03$ & $9.94 \mathrm{E}-01$ \\
\hline-4458.9 & $1.76 \mathrm{E}-02$ & $1.00 \mathrm{E}+00$ & $9.57 \mathrm{E}-04$ & $2.60 \mathrm{E}-03$ & $1.43 \mathrm{E}-02$ & $1.70 \mathrm{E}-02$ & $4.75 \mathrm{E}-08$ & $1.01 \mathrm{E}-09$ & $4.84 \mathrm{E}-03$ & 7.02E-04 & $1.01 \mathrm{E}-09$ & $1.19 \mathrm{E}-08$ & $1.01 \mathrm{E}-09$ & $1.61 \mathrm{E}-03$ & $9.95 \mathrm{E}-01$ \\
\hline-4459.1 & $1.75 \mathrm{E}-02$ & $1.00 \mathrm{E}+00$ & $9.75 \mathrm{E}-04$ & $2.44 \mathrm{E}-03$ & $1.46 \mathrm{E}-02$ & $1.74 \mathrm{E}-02$ & $4.06 \mathrm{E}-08$ & $1.01 \mathrm{E}-09$ & $4.74 \mathrm{E}-03$ & 7.17E-04 & $1.01 \mathrm{E}-09$ & $1.02 \mathrm{E}-08$ & $1.01 \mathrm{E}-09$ & $1.58 \mathrm{E}-03$ & $9.95 \mathrm{E}-01$ \\
\hline-4459.3 & $1.74 \mathrm{E}-02$ & $1.00 \mathrm{E}+00$ & $9.94 \mathrm{E}-04$ & $2.27 \mathrm{E}-03$ & $1.51 \mathrm{E}-02$ & $1.79 \mathrm{E}-02$ & 3.42E-08 & $1.01 \mathrm{E}-09$ & 4.64E-03 & 7.36E-04 & $1.01 \mathrm{E}-09$ & 8.54E-09 & 1.01E-09 & $1.55 \mathrm{E}-03$ & $9.96 \mathrm{E}-01$ \\
\hline-4459.5 & $1.74 \mathrm{E}-02$ & $1.00 \mathrm{E}+00$ & $1.01 \mathrm{E}-03$ & $2.10 \mathrm{E}-03$ & $1.55 \mathrm{E}-02$ & $1.84 \mathrm{E}-02$ & $2.78 \mathrm{E}-08$ & $1.01 \mathrm{E}-09$ & 4.53E-03 & 7.65E-04 & $1.01 \mathrm{E}-09$ & 6.94E-09 & 1.01E-09 & $1.51 \mathrm{E}-03$ & $9.96 \mathrm{E}-01$ \\
\hline-4459.7 & $1.73 \mathrm{E}-02$ & $1.00 \mathrm{E}+00$ & $1.04 \mathrm{E}-03$ & $1.91 \mathrm{E}-03$ & $1.60 \mathrm{E}-02$ & $1.90 \mathrm{E}-02$ & $2.06 \mathrm{E}-08$ & $1.01 \mathrm{E}-09$ & $4.41 \mathrm{E}-03$ & $8.28 \mathrm{E}-04$ & $1.01 \mathrm{E}-09$ & $5.15 \mathrm{E}-09$ & $1.01 \mathrm{E}-09$ & $1.47 \mathrm{E}-03$ & $9.97 \mathrm{E}-01$ \\
\hline-4459.9 & $1.73 \mathrm{E}-02$ & $1.01 \mathrm{E}+00$ & $1.06 \mathrm{E}-03$ & $1.72 \mathrm{E}-03$ & $1.65 \mathrm{E}-02$ & $1.96 \mathrm{E}-02$ & $1.18 \mathrm{E}-08$ & $1.01 \mathrm{E}-09$ & 4.29E-03 & $1.00 \mathrm{E}-03$ & $1.01 \mathrm{E}-09$ & 2.96E-09 & 1.01E-09 & $1.43 \mathrm{E}-03$ & $9.97 \mathrm{E}-01$ \\
\hline-4460.1 & $1.72 \mathrm{E}-02$ & $1.01 \mathrm{E}+00$ & $1.09 \mathrm{E}-03$ & $1.51 \mathrm{E}-03$ & $1.71 \mathrm{E}-02$ & $2.02 \mathrm{E}-02$ & $5.04 \mathrm{E}-09$ & $1.01 \mathrm{E}-09$ & $4.16 \mathrm{E}-03$ & $1.37 \mathrm{E}-03$ & $1.01 \mathrm{E}-09$ & $1.26 \mathrm{E}-09$ & $1.01 \mathrm{E}-09$ & $1.39 \mathrm{E}-03$ & $9.98 \mathrm{E}-01$ \\
\hline-4460.3 & $1.72 \mathrm{E}-02$ & $1.01 \mathrm{E}+00$ & $1.11 \mathrm{E}-03$ & $1.28 \mathrm{E}-03$ & $1.76 \mathrm{E}-02$ & $2.09 \mathrm{E}-02$ & 2.18E-09 & $1.01 \mathrm{E}-09$ & $4.01 \mathrm{E}-03$ & $1.83 \mathrm{E}-03$ & $1.01 \mathrm{E}-09$ & $5.45 \mathrm{E}-10$ & 1.01E-09 & $1.34 \mathrm{E}-03$ & $9.98 \mathrm{E}-01$ \\
\hline
\end{tabular}




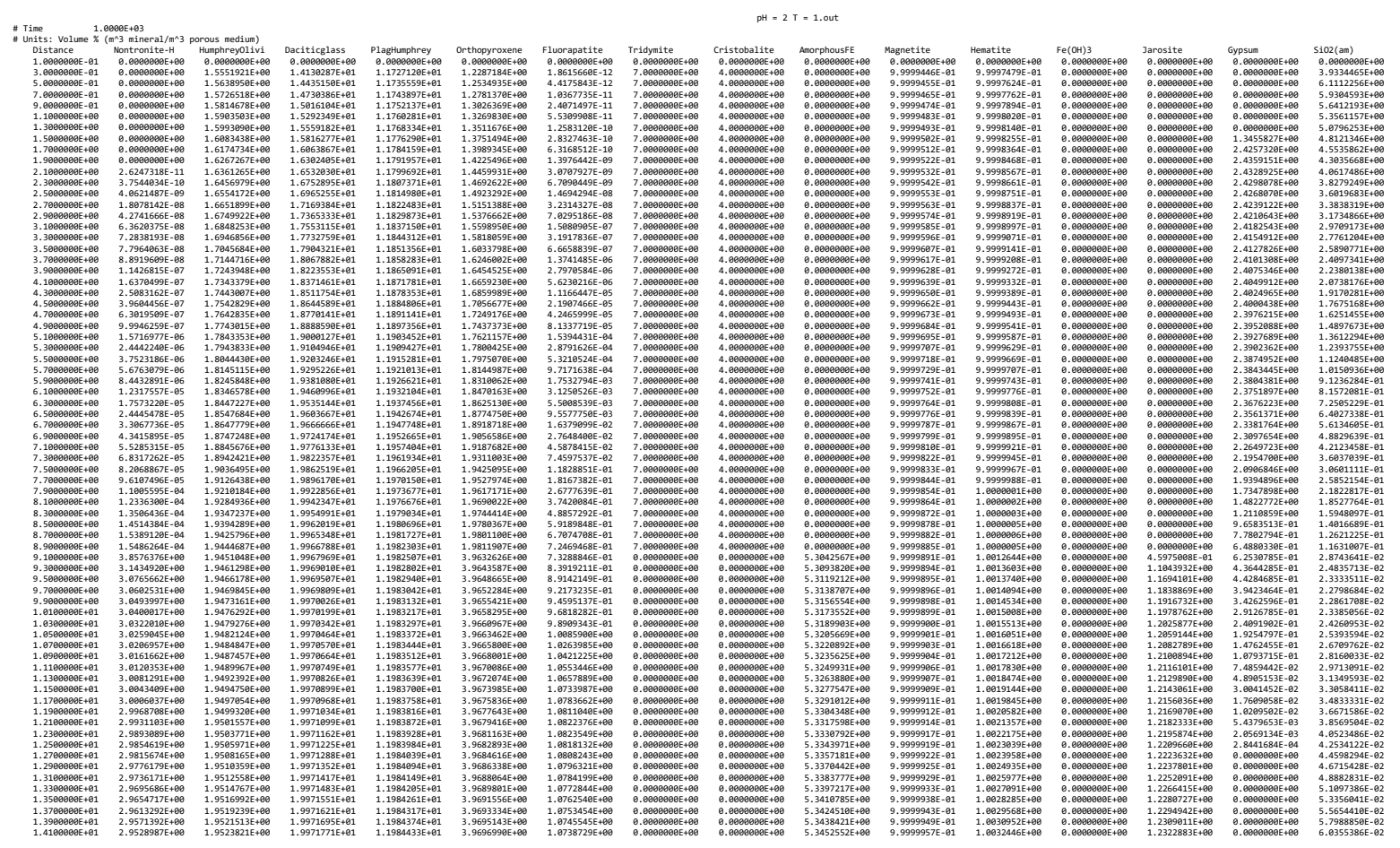




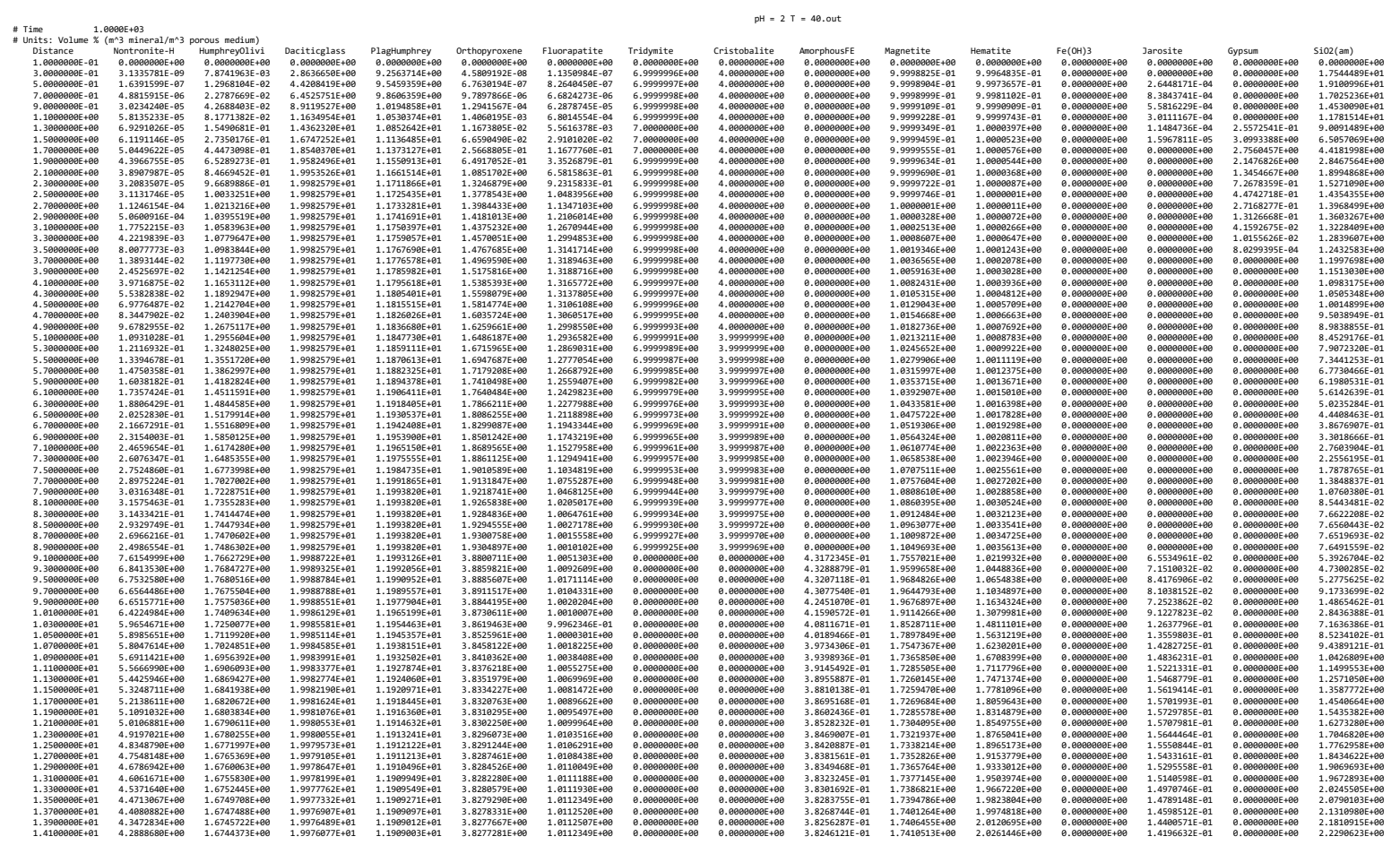




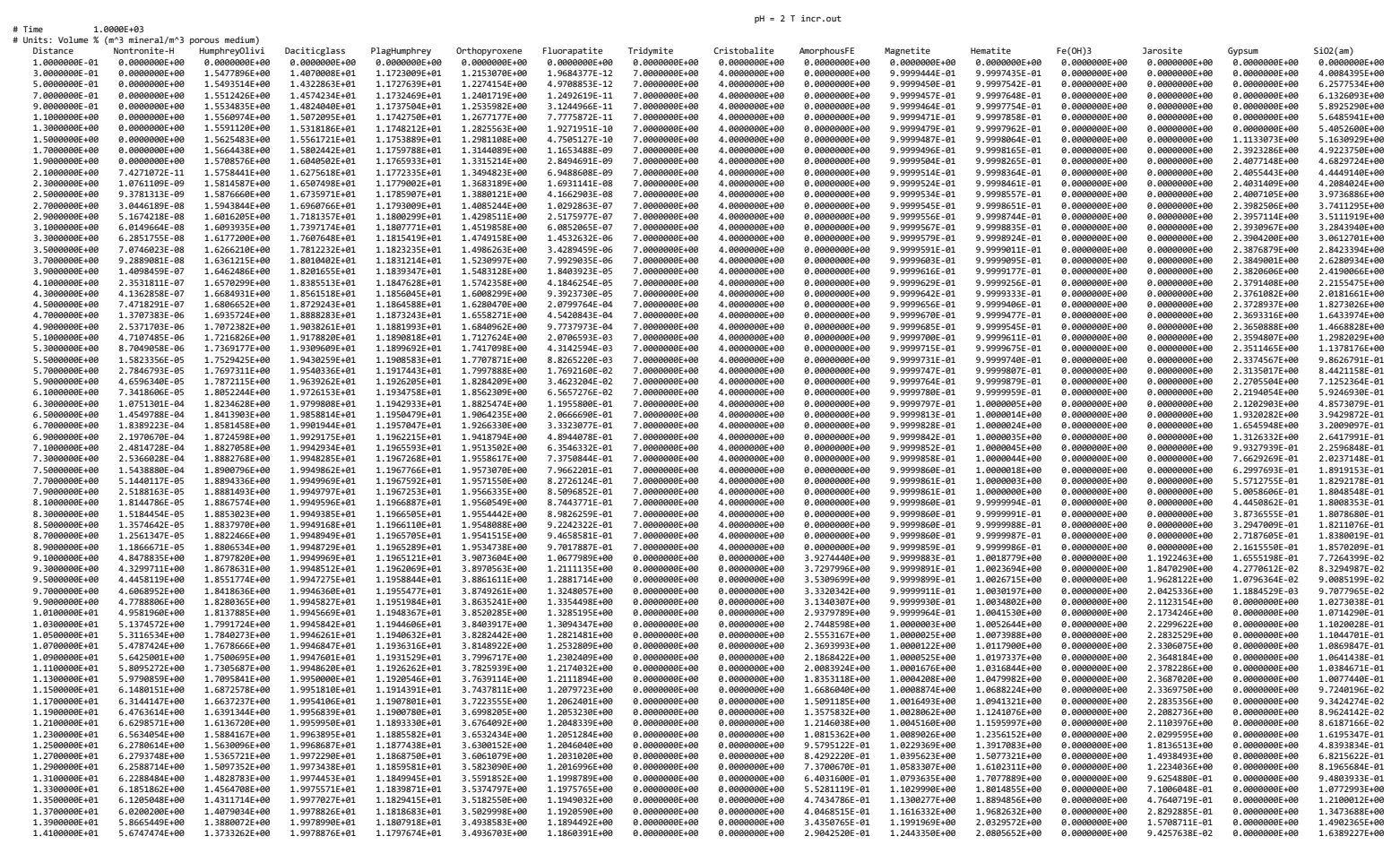




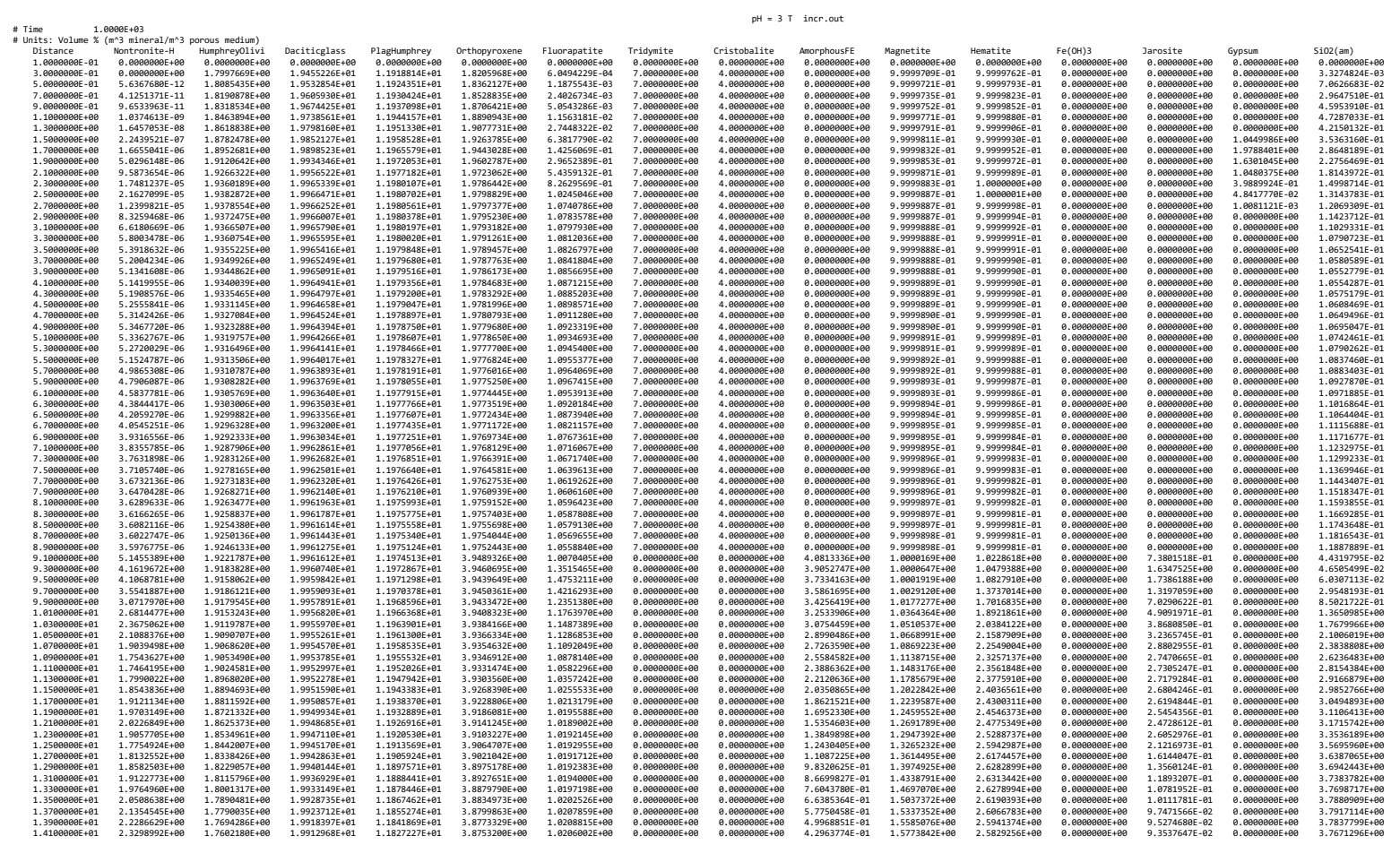




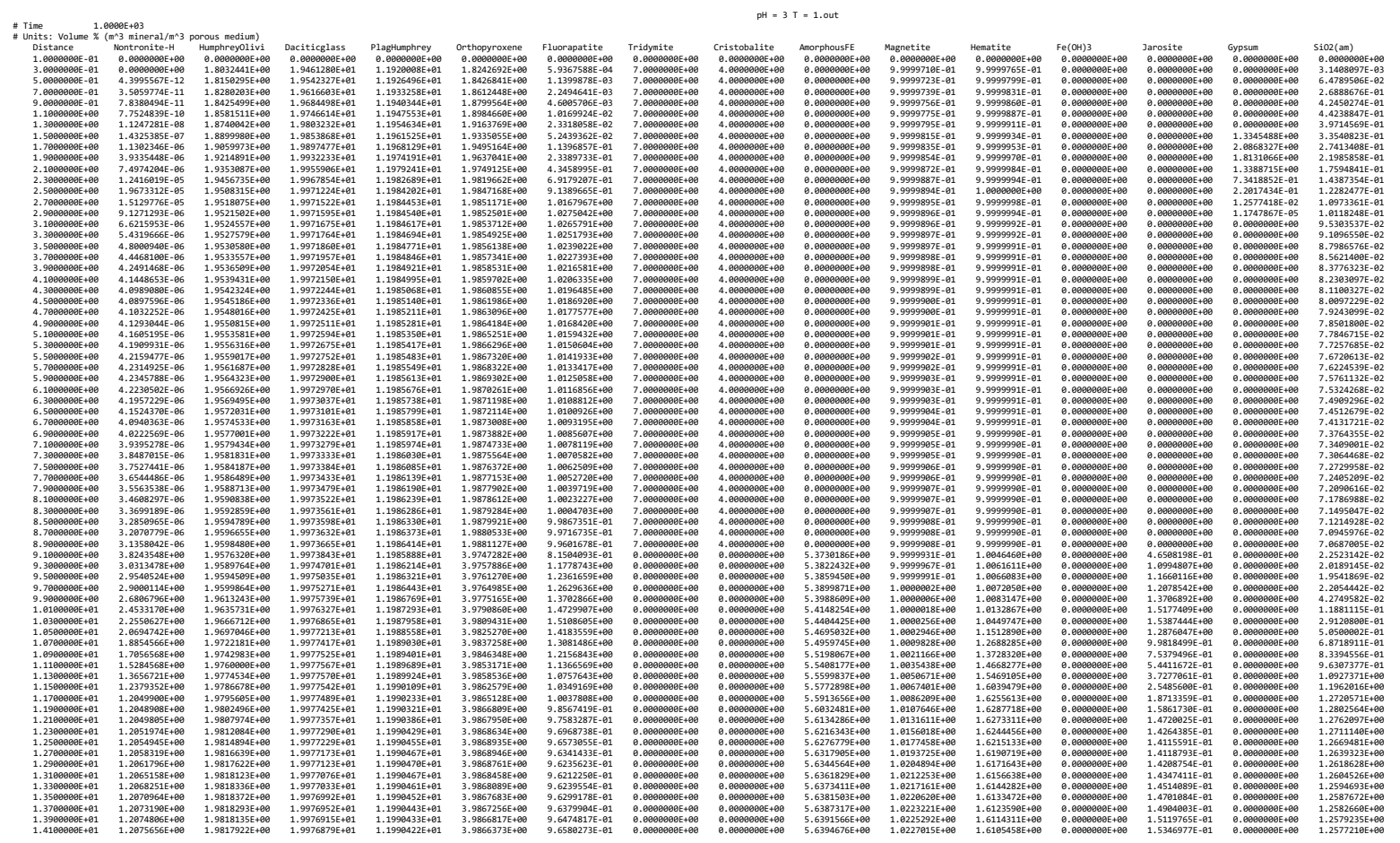




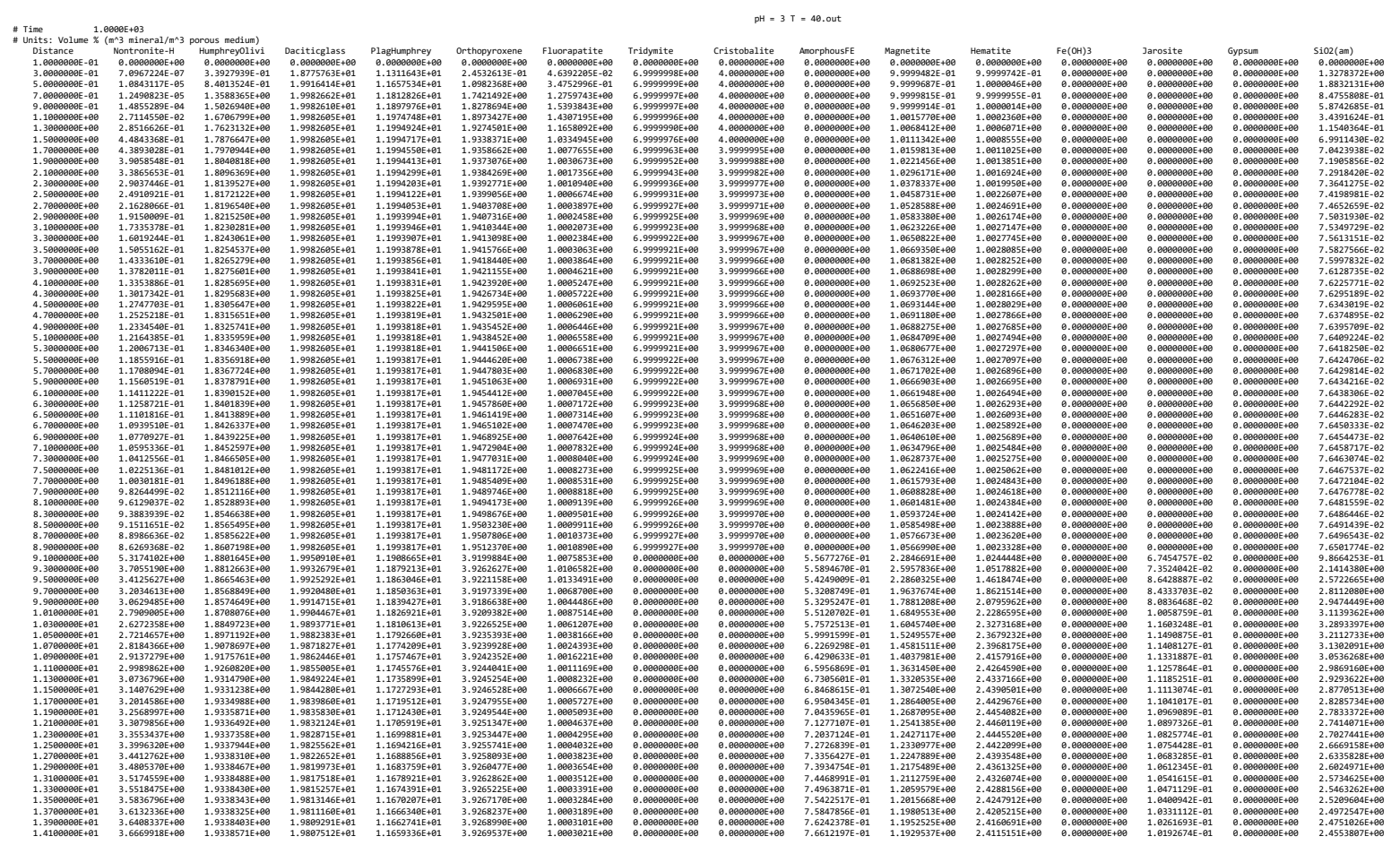




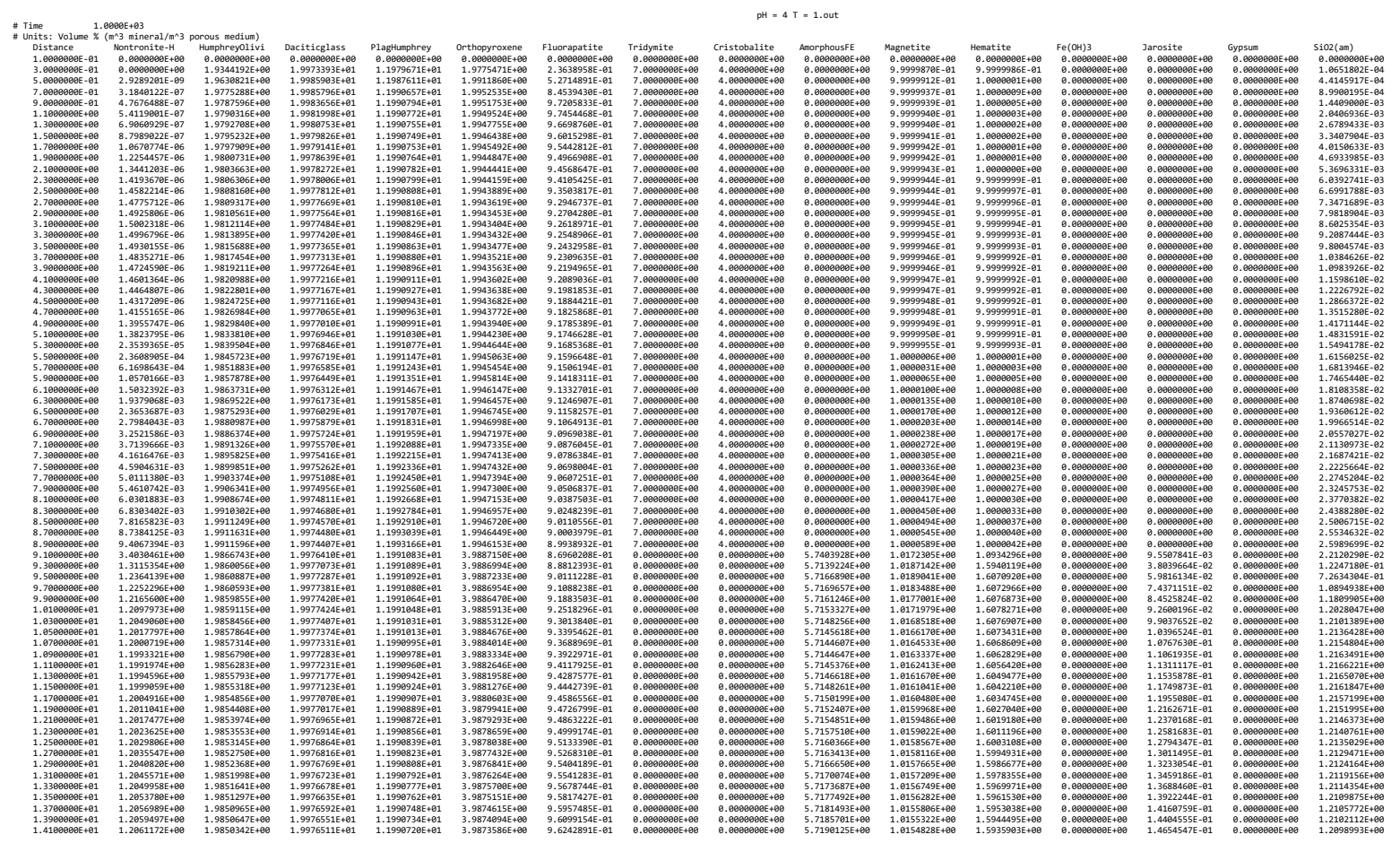




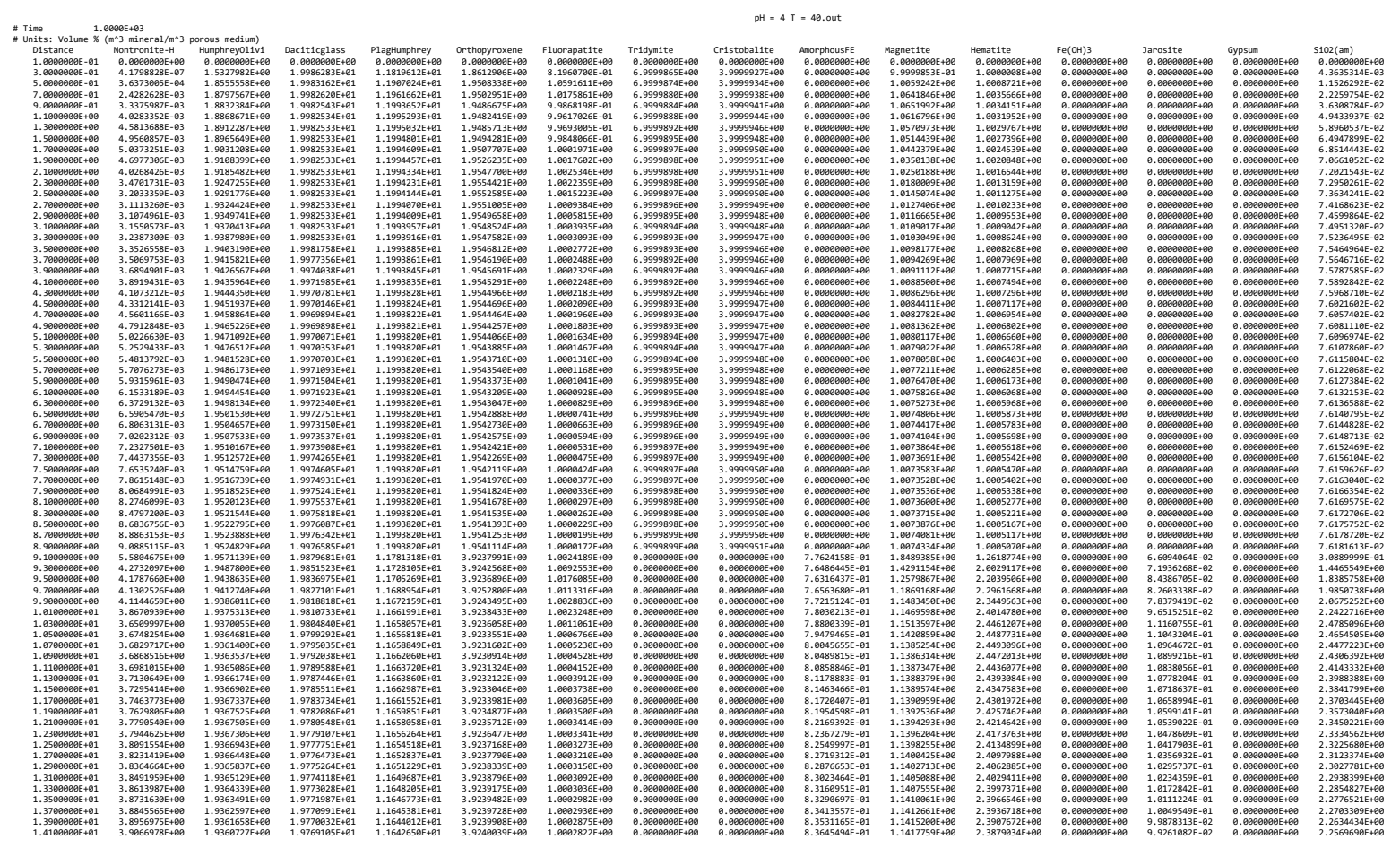




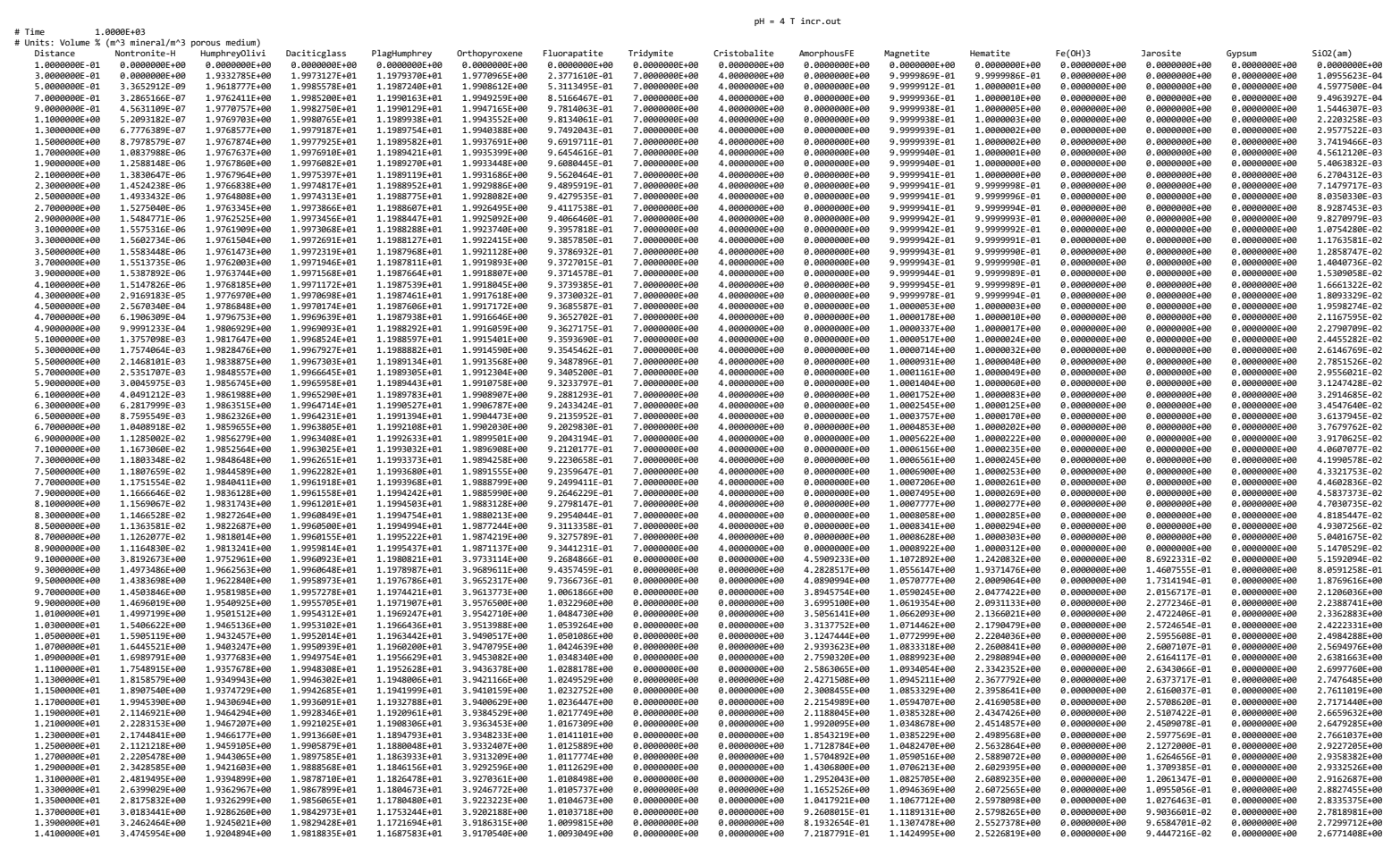




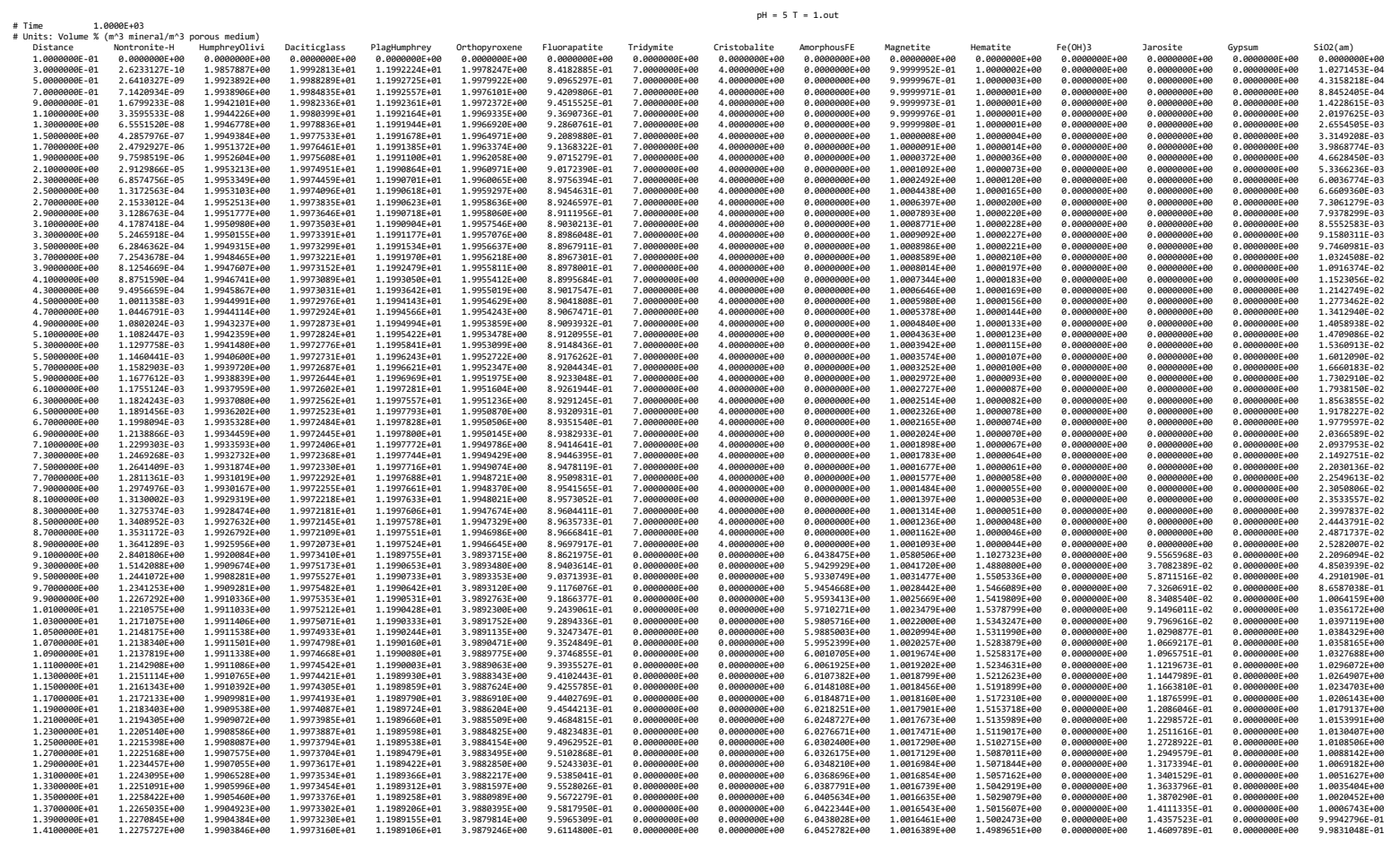




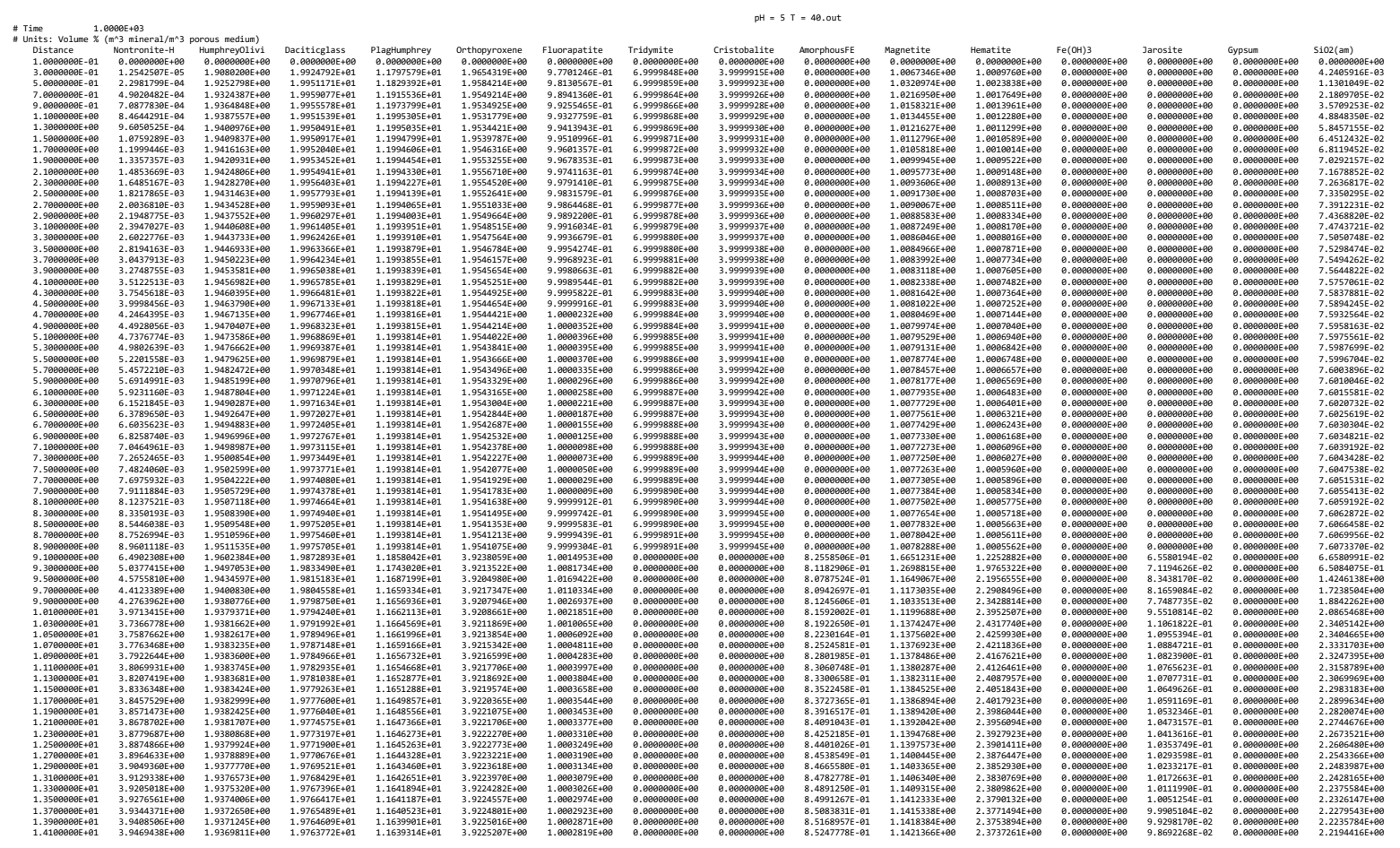




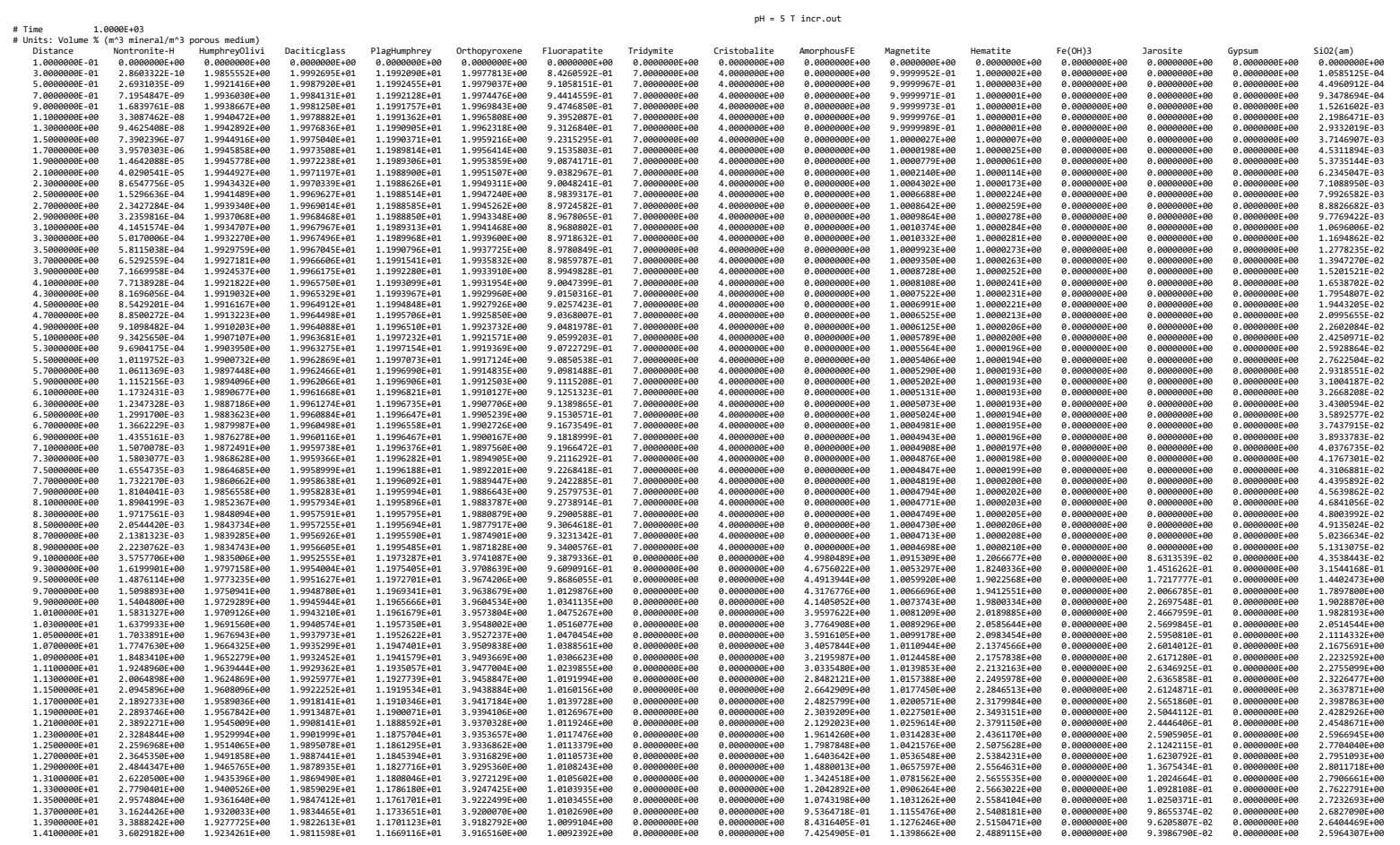




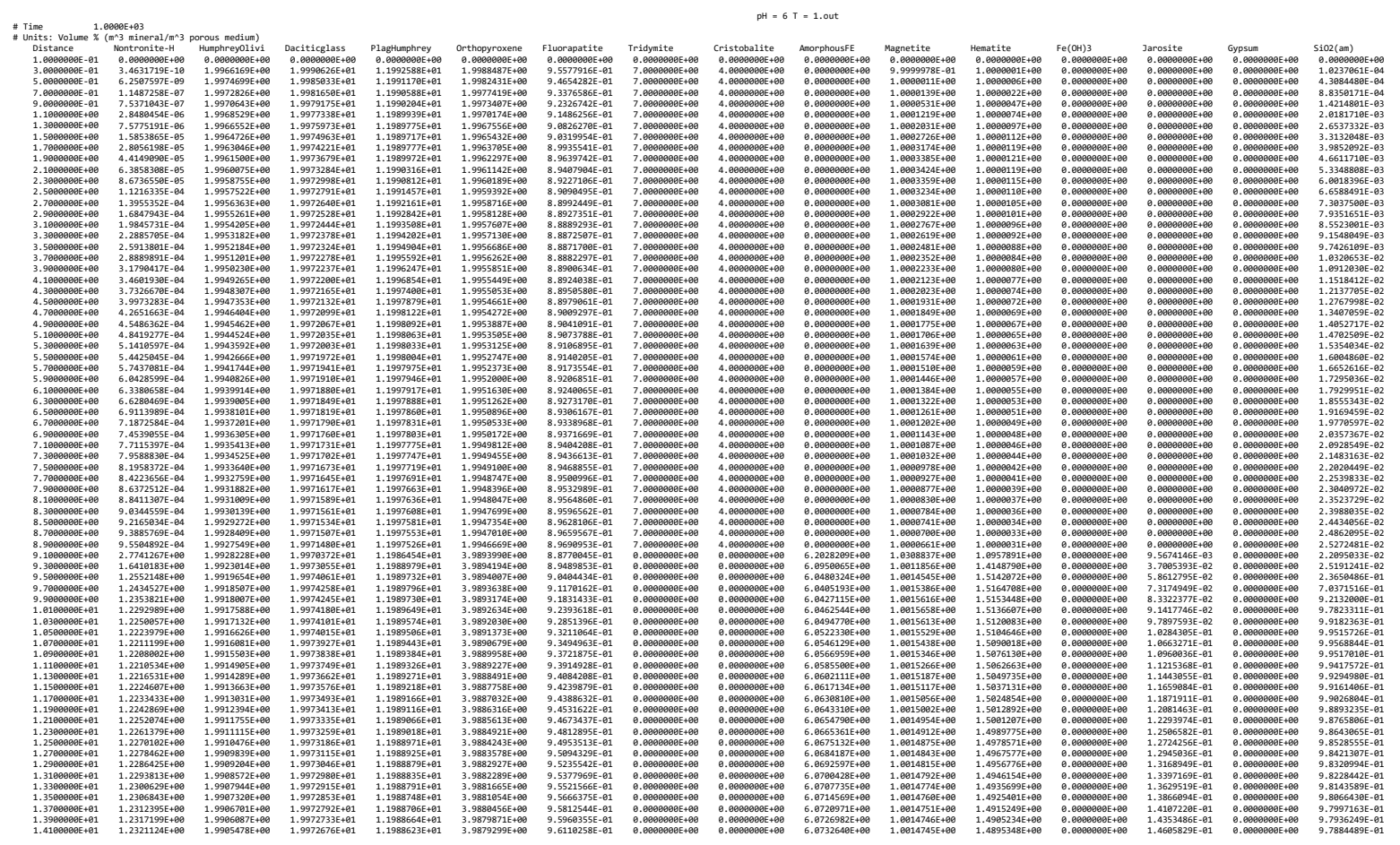




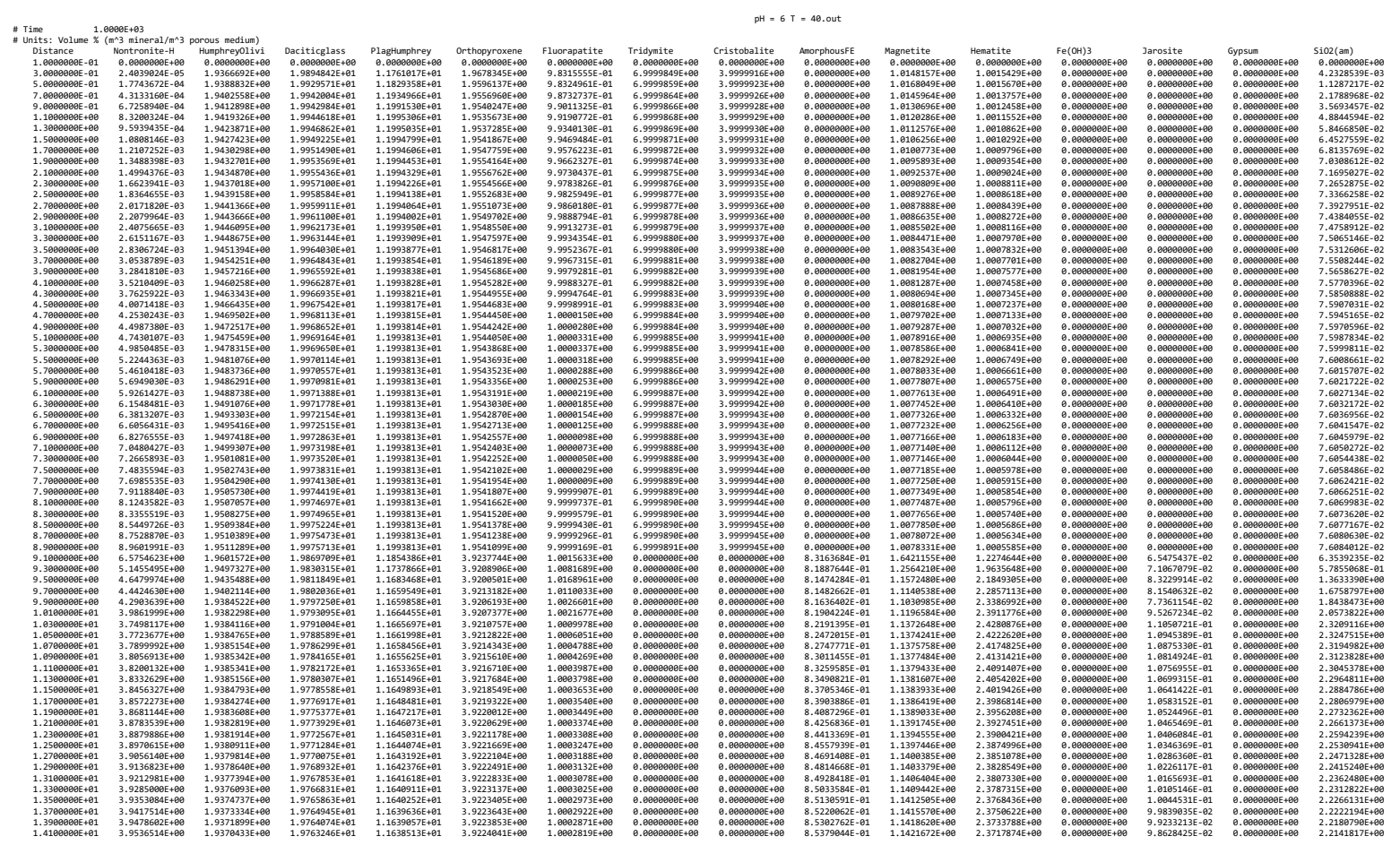




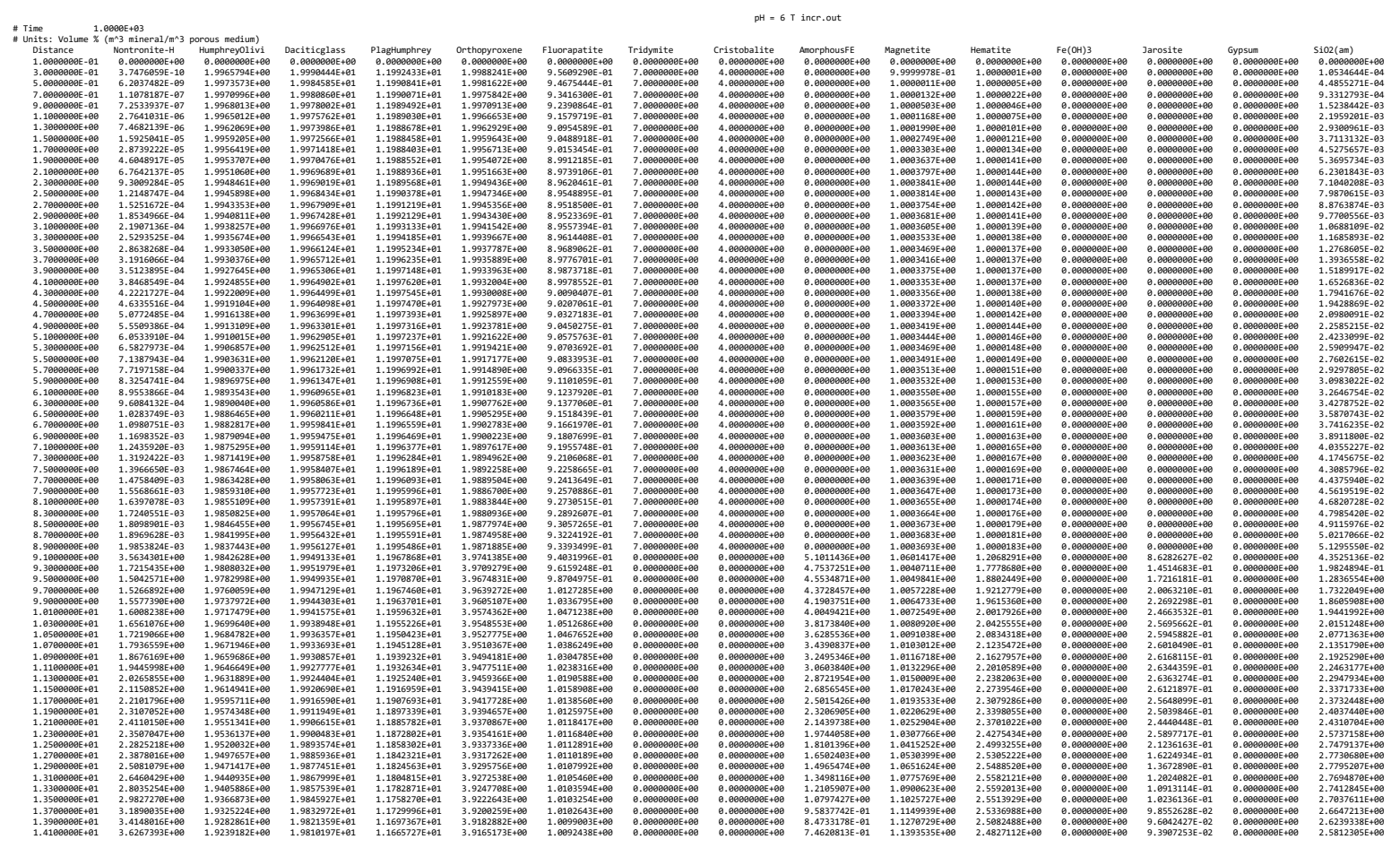




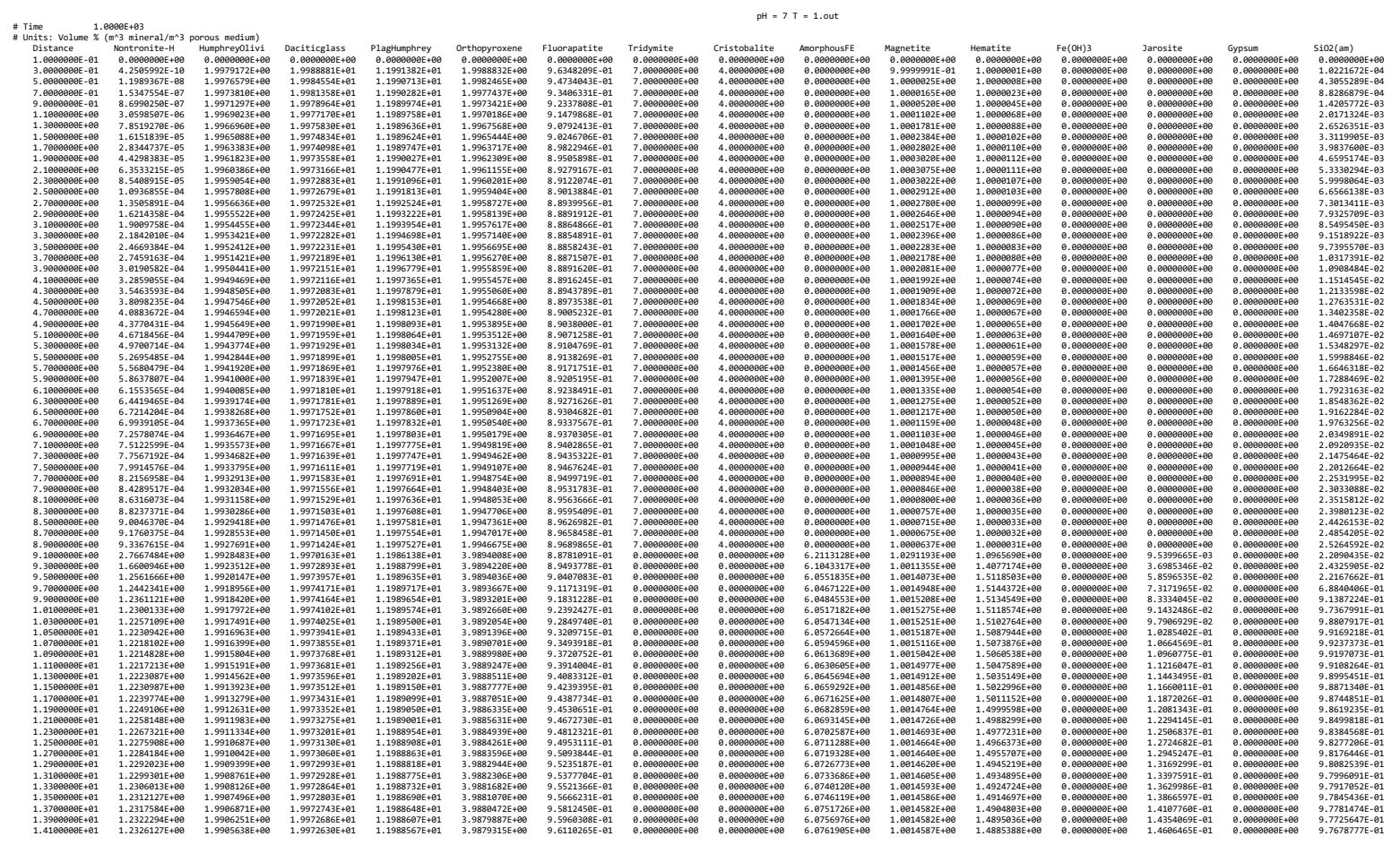




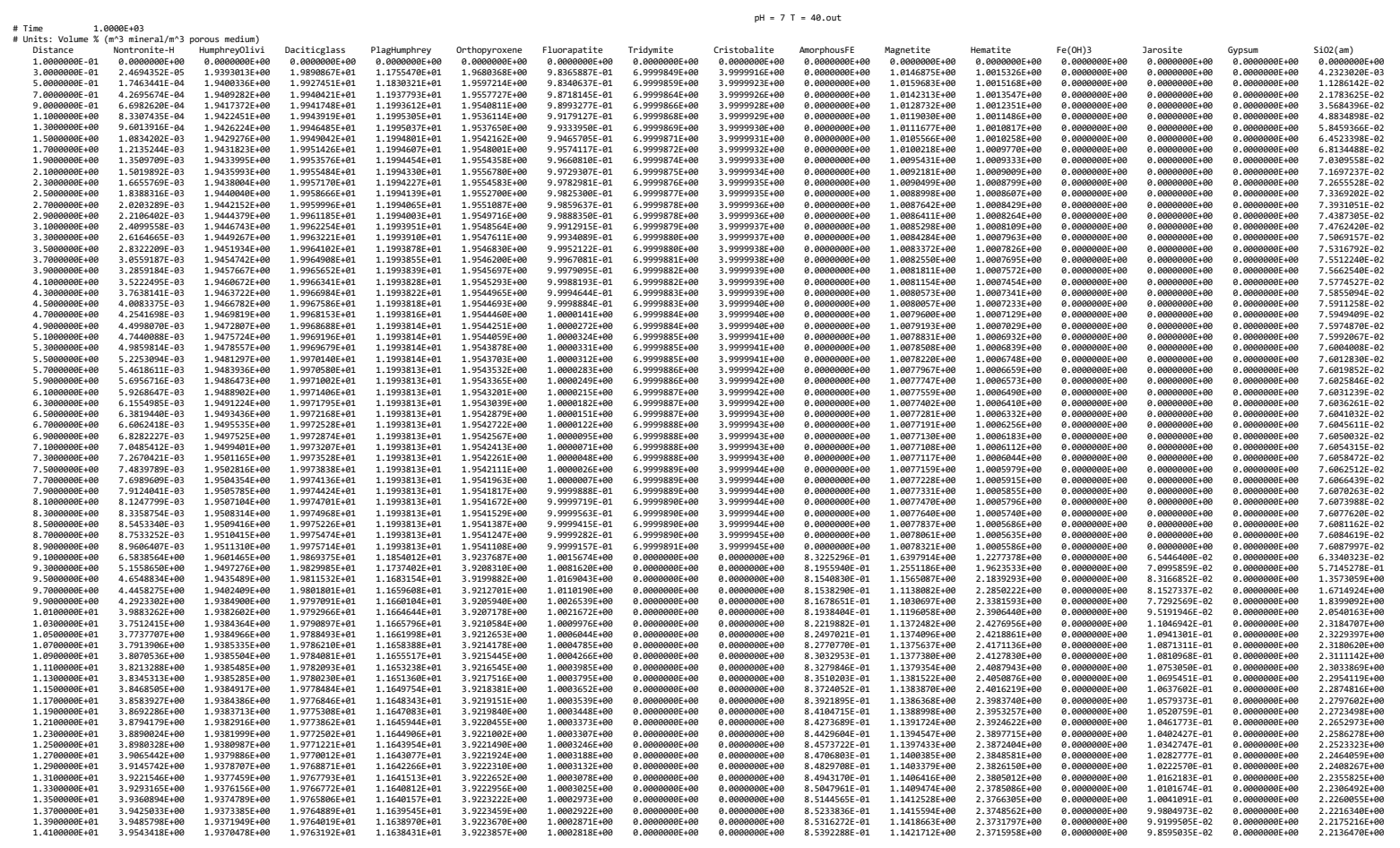




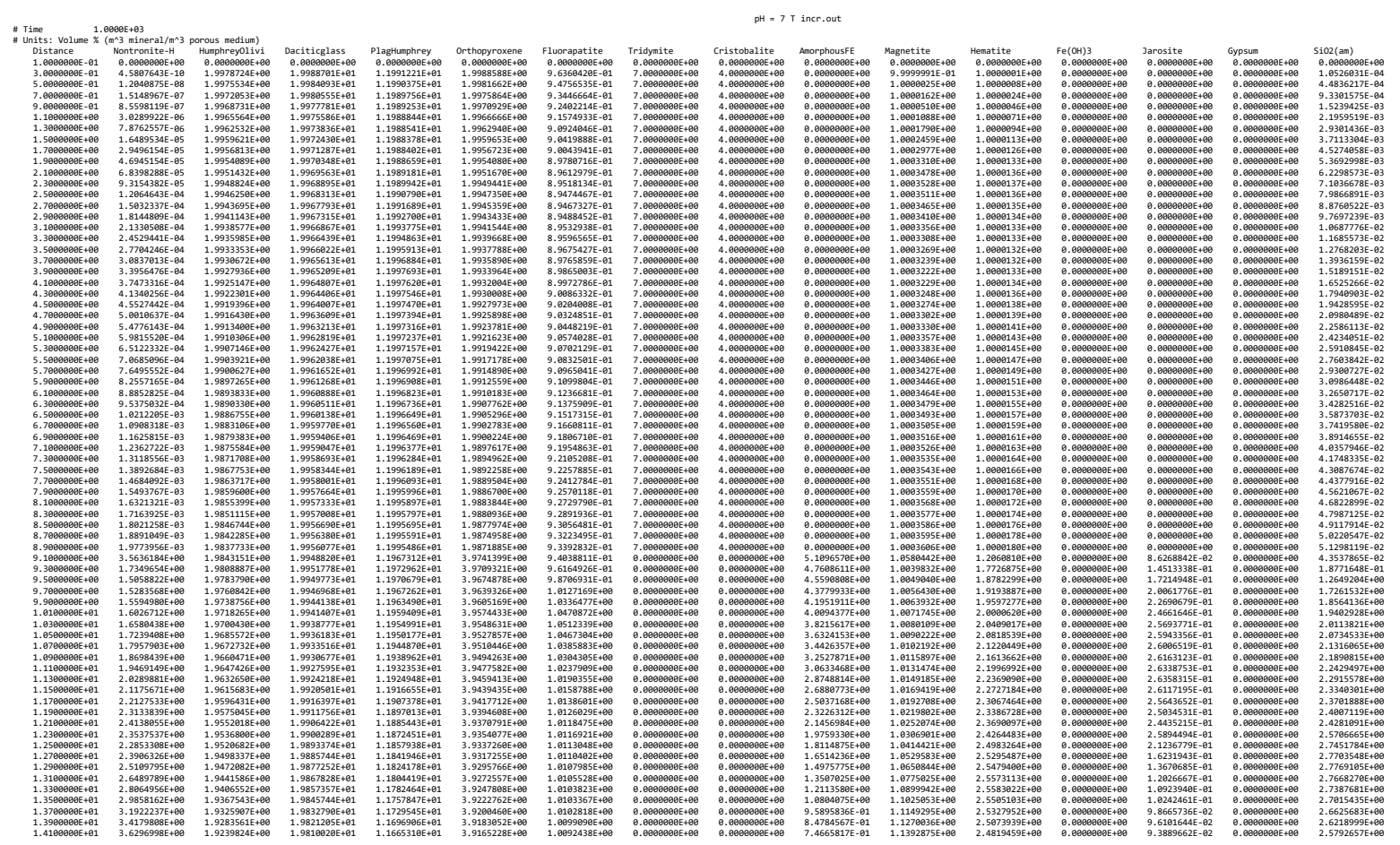




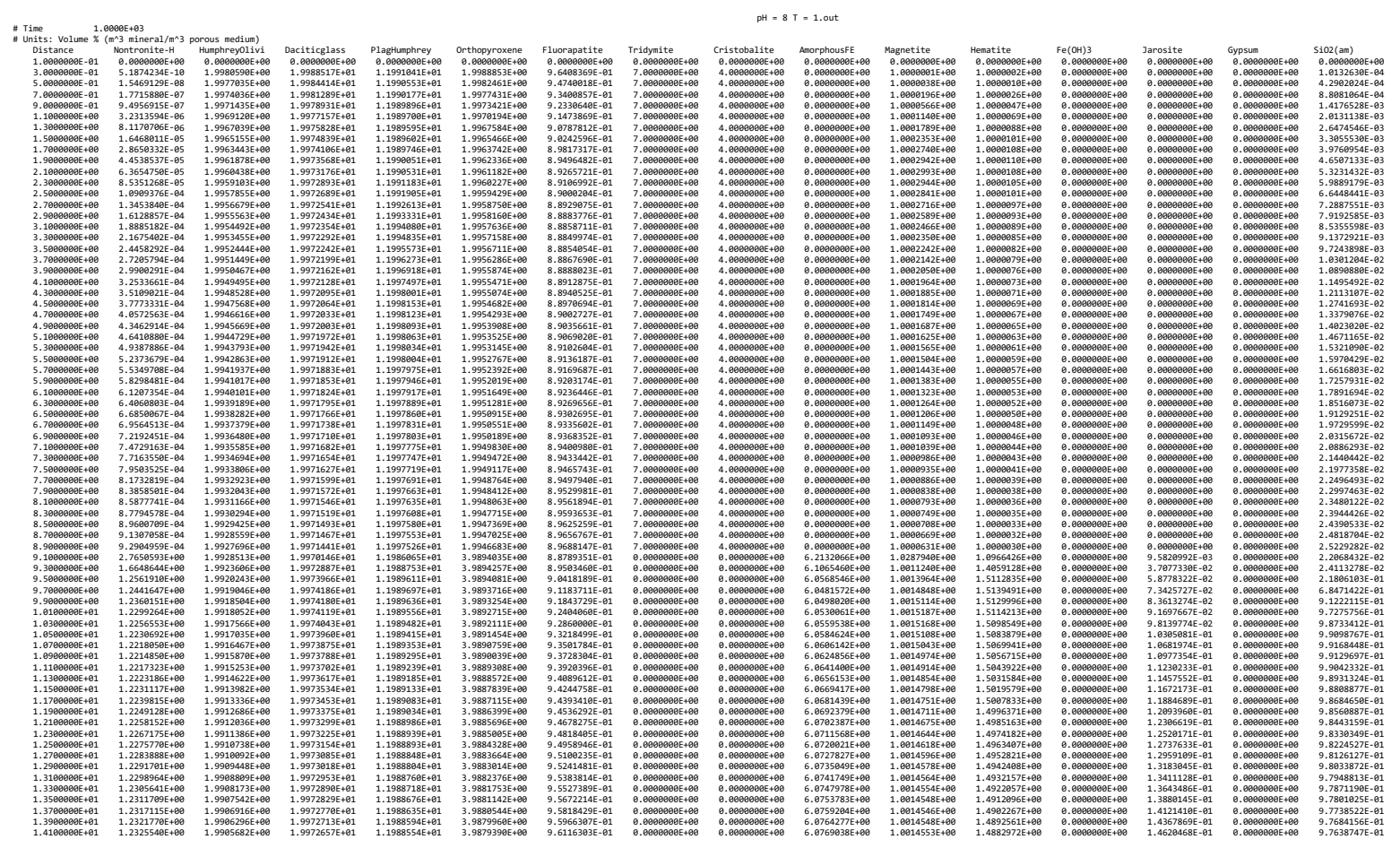




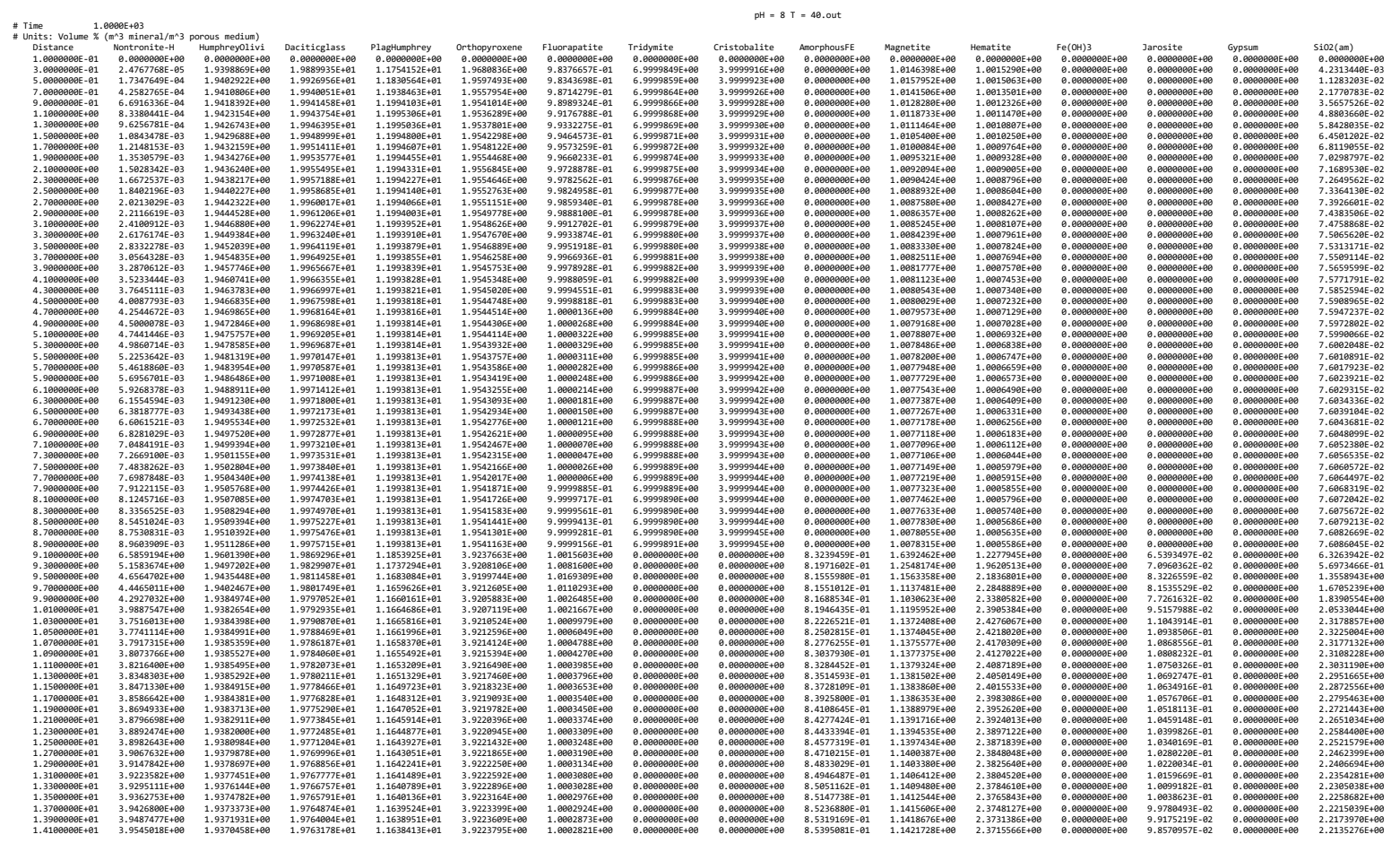




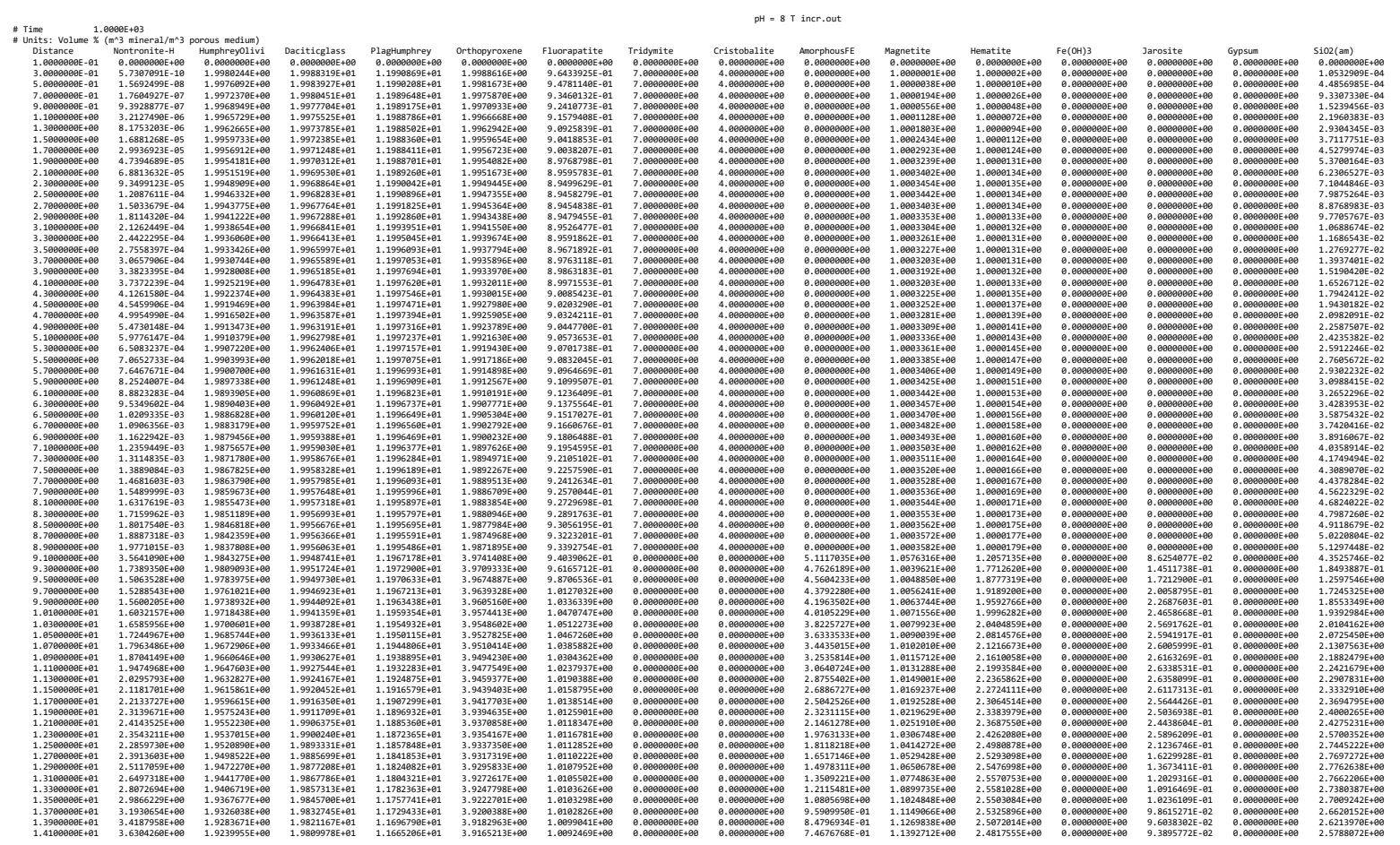

